\title{
Investigation of waterflood front digitations during immiscible displacements in porous media
}

\author{
Matthieu Mascle ${ }^{1, *}$ (D), Elisabeth Rosenberg ${ }^{1}$, Berit Roboele $^{2}$ (D), Espen Kowalewski ${ }^{2}$, and Souhail Youssef ${ }^{1}$ \\ ${ }^{1}$ IFP Energies nouvelles, 1-4, Avenue de Bois-Préau, 92852 Rueil-Malmaison Cedex, France \\ ${ }^{2}$ Equinor ASA, 7005 Trondheim, Norway
}

Received: 12 May 2021 / Accepted: 21 September 2021

\begin{abstract}
In this work, unstable displacements were conducted using special equipment designed to run in-situ CT-scanner experiments. All the displacements were conducted on a homogeneous Bentheimer sandstone plug, of $10 \mathrm{~cm}$ in diameter and $40 \mathrm{~cm}$ in length. Digitations (or fingering) have been observed under varying conditions of injection flowrate, displaced fluid viscosity, and core wettability. They have been characterized at both the core scale, using the core average oil saturation and the water breakthrough; and at the local scale, using the local saturations and had-hoc image processing analysis. It was found that the effect of the different flowing conditions on the front digitations could not be interpreted independently. The oil recovery at brine breakthrough showed a good correlation with the viscous fingering number for the water-wet case. However, a different scaling was observed for the oil-wet case. The interplay of the different flowing conditions mitigates the possibility of constructing a unique scaling number to account for all experimental condition. The local saturation monitoring has provided a new insight to characterize the finger shapes and analyze the production mechanisms. It allowed to distinguish two independent contributions to early breakthrough: viscous dominated digitations and capillary dominated digitations. A two-phases diagram has been constructed to plot and compare these contributions for all flowing conditions. Their evolutions show the main production mechanisms during the flooding. We observed that the viscous digitations were not causing phase trapping at core scale: the core is completely swept after breakthrough. For the water-wet case, we found that the local oil recovery of swept zone remained constant before and after breakthrough while for the oil-wet case it is improving during all the water flooding process.
\end{abstract}

\section{Introduction}

Unstable displacements in porous media can occur when a fluid is displaced by any other fluid of different nature. They can be triggered by a viscosity contrast, a density contrast or capillary forces when the two considered fluids are immiscible. More generally, unstable displacement is expected to occur when the displacing fluid is more mobile than the displaced fluid [1]. They refer to all displacements that are not piston-like. These complex flows lead to poor fluids mixing or sweeping efficiency and early displacing fluid BreakThrough (BT). They have been discussed in an extensive number of publications of various engineering fields [2-6].

When the displacing and displaced fluids are immiscible, the front digitations take the form of fingerings. Their shape and magnitude are governed by various parameters, including the fluids and rock properties and structure, the injection conditions as well as the system dimensions and

\footnotetext{
* Corresponding author: matthieu.mascle@ifpen.fr
}

heterogeneities. Despite their complexity and diversity, unstable displacements are generally characterized using a simple descriptor: the volume of fluids injected until it breaks through. This quantity is referred as the breakthrough earliness. This simple characterization of digitations is partially explained by the difficulty to observe the displacement itself in a porous media. In the literature, many authors have tried to identify the most relevant parameters to correlate the breakthrough earliness using linear stability analysis $[4,5,7,8]$ resulting in numerous dimensionless scaling parameters aiming to account for all these contributions. Among the most referred to, Peters and Flock [8] have constructed a dimensionless number (referred to here as the $I_{\text {sc }}$ number) using Chuoke's stability theory [9] to predict the onset of front digitations. Later, Lenormand et al. [10] proposed a phases-diagram using two dimensionless numbers to dissociate two separated contributions to early breakthrough: the capillary and the viscous fingerings. More recently, Doorwar [11] combined Lenormand's dimensionless numbers and added a tertiary contribution to account for the core dimensions and petrophysical properties. 
The resulting dimensionless number (referred to here as the $N_{I}$ number) has demonstrated good results to predict total fluids recovery at BT (BTR) for water-wet porous media (sandstone rock). Yet, it provides no forecast for fluids fingering shape. Unstable displacements have been extensively studied for water-wet rocks [12] but in a lesser extent for intermediate or oil-wet rock $[8,13-15]$. The few results in the literature show that the scaling obtained for the waterwet case is not valid when the wettability is changed. The wettability, generally well understood at pore-scale, is suffering upscaling issues at core scale. In Peters and Flock's work, the $I_{\mathrm{sc}}$ number demonstrated two different scalings for for the oil-wet and water-wet cases. Thus, constructing a macroscopic descriptor that takes into consideration the core wettability is less trivial. At best, it is considered through the relative permeabilities or the contact angle. This description fails to account for the complex effects of capillary pressure in a porous media.

Fingering has been observed to occur at all scales ranging from the phases by-pass at pore level [16] to the phases partitioning at reservoir level, causing severe early waterbreakthrough. The magnitude of their dimensions variation causes its modelling to be a perilous task using Darcy's scale models [17]. A possible alternative is to upscale flow dynamics properties from laboratory experiments, to account for the complexity of these flows at higher scale resolution [18]. In recent works, several authors [13, 19, 20] have used and adapted Fayers' phenomenological approach of viscous fingering [21] to upscale relative-permeabilities. These models, referred to as lumped-finger, rely on the merging of all fingering in a unique finger, described by various physical parameters. This approach allows to obtain a satisfying match of the global experimental data. Yet, the local saturations and the equivalent finger shape is rarely studied. The phenomenological modelling demonstrates the necessity to have a $2 \mathrm{D}$ or $3 \mathrm{D}$ flow visualization to characterize front digitations forms. Glass micro-models have proven their usefulness for visual inspection. Still they are by nature limited in dimensions and connectivity [20,22]. Slab experiments conducted on Bentheimer samples have allowed to look further in the 2D fingerings characterization $[17,23]$. However, the core width is restricted with these experiments, leading to high dimensions ratio that strongly affect the fingers growth as demonstrated by Doorwar and Mohanty in [24].

The objective of the present article is to show and use all the potential of the CT-scan imaging to describe and characterize the front chap in immiscible displacement. We will therefore further investigate the effect of different parameters namely viscosity ratio, displacing fluid velocity, and wettability on the evolution of mean and local saturation as a function of injected pore volume. We will also focus on the dependency of saturation at breakthrough to flowrate and viscosity ration using dimensionless number. To achieve this goal a set of immiscible displacements were conducted using special equipment designed to run CT-scanner in-situ core flooding on full-size sample. In the first section we present the experimental procedure used in this work as well as data processing method used to interpret 3D CT-scan images. Results are then presented and

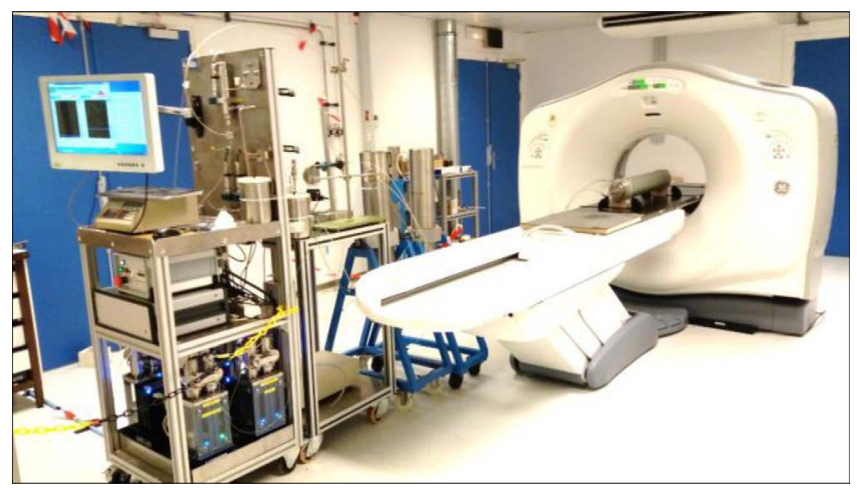

Fig. 1. Overview of the IFPEN medical CT Scan GEHC Discovery $750 \mathrm{HD}$ and the experimental setup.

examined to relate experimental observations to operating conditions. The access to real-time $3 \mathrm{D}$ saturation distribution naturally provides more information as they include the special and temporal behavior of the transport. Consequently, we gain more insight on the front displacement behavior.

\section{Material and methods}

\subsection{Coreflood set-up}

Experiments were conducted using a special equipment designed to run in-situ experiments under CT-scan. An overview of the experimental set-up is given in Figure 1. The equipment is composed of an X-ray transparent core holder and a mobile rig able to inject different fluids at up to 100 bar. The core holder is designed to handle cylindrical samples of $10 \mathrm{~cm}$ in diameter and up to $60 \mathrm{~cm}$ in length. The core holder body is made of a $5 \mathrm{~mm}$ thickness aluminum alloy which allows good X-ray transmission at an energy of $140 \mathrm{kVP}$.

A multi-pump system is used, allowing to inject different fluids without need of changing pump fluid and thus avoid fluid pollution. The low viscosity fluids are injected using Vindum VP-12 K pumps. The high viscosity fluids are injected using $5 \mathrm{~L}$ piston cylinders, connected to a Vindum pump to drive the pistons. The Back PRessure (BPR) is regulated to 20 bars. The confining pressure is imposed to 50 bars using an Isco syringe pump, fed with deionized water (MilliQ). Experiments were conducted at scanner room temperature, regulated by an air conditioner.

The differential pressure is monitored using two ABB differential transducer on 2.5 bar and 25 bar in parallel as well as 150 bar Keller absolute pressure transducers connected to the upstream and downstream static fluids lines. The fluids saturation is monitored using a medical CT-scanner GEHC Discovery 750 HD dual energy. It is operated at $140 \mathrm{kVP}$ with a beam current of $260 \mathrm{~mA}$ to minimize signal/noise ratio. This equipment imposes the coreflood cell to be installed horizontally. The produced effluents are collected and gravity-separated in a vertical graduated burette to support the saturation computation. 
Table 1. Core dimensions and petrophysical properties.

\begin{tabular}{lc}
\hline Dimensions $(D, L)$ & $10 \mathrm{~cm}, 40 \mathrm{~cm}$ \\
Mean porosity $\Phi$ & $22.2 \%$ \\
Pore-Volume $(\mathrm{PV})$ & $722 \mathrm{cc}$ \\
Absolute permeability $K$ & $2530 \mathrm{mD}$ \\
Dispersion $D$ & $5.4 \times 10^{-9} \mathrm{~m}^{2} / \mathrm{s}$ \\
\hline
\end{tabular}

\subsection{Rock characterization}

A unique $10 \mathrm{~cm}$ in diameter and $40 \mathrm{~cm}$ long water-wet Bentheimer core is used for all coreflood experiments (Tab. 1). The core is analyzed with the dual energy mode of GEHC called GSI (Gemstone Spectral Imaging) to assess the rock homogeneity (Fig. 2). It suggests a slight density increase from left to right (from inlet to outlet). A small heterogeneity shaped as a $1 \mathrm{~cm}$ radius sphere is visible near the center of the core. This centimeter-scale heterogeneity won't impact the flooding experiments. Still, it shows the Bentheimer rock can exhibit some heterogeneities. The mean porosity is estimated to $22.2 \%$, giving a core Pore Volume (PV) of $722 \mathrm{cc}$. The porosity profile is given in Figure 2. It shows the small porosity reduction of $2 \%$ toward the core outlet. The core permeability $K$ is measured with the brine at a confining pressure of 50 bar and a pore pressure of 20 bar. It is estimated to $2530 \mathrm{mD}$.

A Mercury Intrusion Capillary Pressure (MICP) has been run on a twin Bentheimer rock sample (permeability estimated to 2 Darcy). The capillary pressure curve (for drainage displacement) is estimated for our fluids and rock system considering an IFT of $45 \mathrm{mN} / \mathrm{m}$ (see Tab. 2) and a contact angle of $0{ }^{\circ} \mathrm{C}$. It is showed in Figure 3A, the throat radius distribution is computed from the MICP and showed in Figure 3B, showing a single mode distribution centered around $20 \mu \mathrm{m}$. The Capillary Pressure curve (Pc curve) obtained matches other measurements from the literature obtained on Bentheimer rocks [25, 26]. For the drainage displacement, it shows an average capillary pressure of $50 \mathrm{mBars}$ for the saturations where the fluids are both mobile (for $S w$ ranging from $20 \%$ to $80 \%$ ). As the work presented here focuses on imbibition displacement mainly, we need to estimate the Pc curve for this displacement. In the work of Raeesi et al. [25], the Pc curve measured during imbibition on the Bentheimer sample shows $\mathrm{Pc}_{\mathrm{c}}$ values around 25 mBars for brine saturations between $40 \%$ and $60 \%$. These values are several orders of magnitude lower than the differential pressures that have been recorded during the waterflood displacements. From this observation, the capillary pressure will be assumed to be null during our waterflood displacements.

The core dispersivity is assessed using a tracer injection test. A brine water is injected at $1.4 \mathrm{cc} / \mathrm{min}$ in the core, initially saturated with deionized water. The normalized tracer concentration is monitored using the CT-scanner. The concentration profiles measured during the tracer injection are displayed in Figure 4, with local tracer saturations displayed on a sagittal slice (see Sect. 2.4). The profiles and the local saturations show a fairly homogenous sweeping, with a limited tracer dispersion. Using the
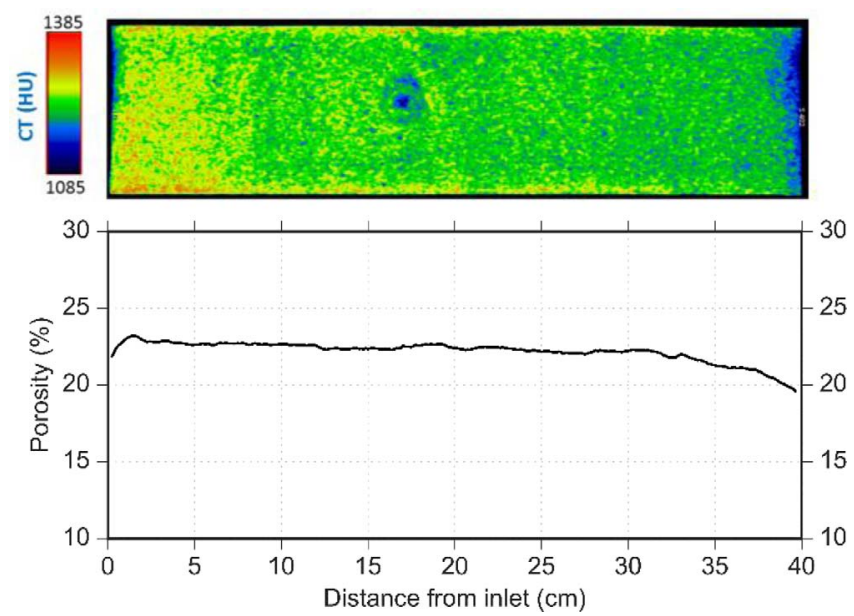

Fig. 2. CT Sagittal cross section rendering and core porosity profile.

standard analytical solution [27], the core dispersion is fitted with $D=5.4 \times 10^{-9} \mathrm{~m}^{2} / \mathrm{s}(c f$. Fig. 5) which corresponds to a dispersivity of $2 \mathrm{~mm}$. The measured magnitude of dispersivity indicates that the core is overall homogenous. The homogeneity of the sample is an important condition in this study to mitigate its effect on the front displacement [28].

The first set of experiments (5 waterflood displacements) was conducted with the core's originate water-wet wettability preserved. In the second set of experiments (3 waterflood displacements), the core was first aged by circulating crude oil at low flowrate $(1.5 \mathrm{cc} / \mathrm{h})$ for 20 days at a temperature of $70{ }^{\circ} \mathrm{C}$. The core was initially set to an Swi of $11.1 \%$. The objective of aging is to alter the originate core wettability toward a more oil-wet condition.

\subsection{Fluids}

Experiments have been conducted using a $70 \mathrm{~g} / \mathrm{L}$ TDS brine. For the water-wet displacements, three mineral oils have been used: Isopar L, Primol, Drakeol. Their viscosities are estimated to $5 \mathrm{cP}, 170 \mathrm{cP}$ and $320 \mathrm{cP}$ respectively at $19.5{ }^{\circ} \mathrm{C}$. After ageing the core, a Dilute Crude-Oil (DC-O) has been used, to preserve the core altered wettability. The DC-O is obtained by mixing the Crude-Oil (C-O) used for the core ageing with cyclohexane $(22 \% \mathrm{wt})$. The dilution aims to reduce its viscosity, allowing working at ambient temperature. For the corefloods conducted with the DC-O, the scanner room was regulated to $17.8^{\circ} \mathrm{C}$. The DC-O viscosity is estimated to $540 \mathrm{cP}$ at this temperature. All oils viscosities are estimated from experimental data. A standard error of $\delta T=1{ }^{\circ} \mathrm{C}$ is considered for the temperature regulation. It is used to compute the standard error $\delta \mu$ for each oil viscosity. All values are summarized in Table 2.

The brine water used for the water flooding is a mixture of $\mathrm{NaCl}(30 \mathrm{~g} / \mathrm{L})$ doped with $\mathrm{NaI}(40 \mathrm{~g} / \mathrm{L})$ to increase the $\mathrm{CT}$ contrast. Its viscosity is estimated using tabulated data to $1.18 \mathrm{cP}$ at $19.5^{\circ} \mathrm{C}$ and $1.23 \mathrm{cP}$ at $17.8^{\circ} \mathrm{C}$. The InterFacial Tension (IFT) of the mineral oils and the DC-O in 
Table 2. Experimental corefloods design, with the $I_{\mathrm{sc}}$ number computed (see Eq. (1)).

\begin{tabular}{|c|c|c|c|c|c|c|c|}
\hline Coreflood & $\begin{array}{c}\text { Core } \\
\text { treatment }\end{array}$ & Oil & $\begin{array}{c}\text { Oil viscosity }(\mathrm{cP}) / \\
\text { oil standard error }(\mathrm{cP}) \\
\end{array}$ & $\begin{array}{c}\text { IFT } \\
(\mathrm{mN} / \mathrm{m}) \\
\end{array}$ & $\begin{array}{c}\text { Injection flowrate } \\
(\mathrm{cc} / \mathrm{min})\end{array}$ & $I_{\mathrm{sc}}$ & $N_{g}$ \\
\hline CF1 & \multirow{5}{*}{$\begin{array}{l}\text { No treatment } \\
\text { (water-wet) }\end{array}$} & Isopar L. & $5(\delta \mu=3)$ & 45 & 7.0 & 3 & 0.09 \\
\hline CF2 & & Primol & $170(\delta \mu=13)$ & 30 & 1.4 & 50 & 0.30 \\
\hline CF3 & & & & & 7.0 & 250 & 0.06 \\
\hline CF4 & & Drakeol & $320(\delta \mu=26)$ & 51 & 1.4 & 50 & 0.30 \\
\hline CF5 & & & & & 7.0 & 280 & 0.06 \\
\hline CF6 & Aged core (oil-wet) & DC-O & $540(\delta \mu=58)$ & 21 & 7.0 & 290000 & 0.06 \\
\hline CF7 & & & & & 0.06 & 2500 & 7.0 \\
\hline $\mathrm{CF} 8$ & & & & & 1.4 & 58500 & 0.30 \\
\hline
\end{tabular}
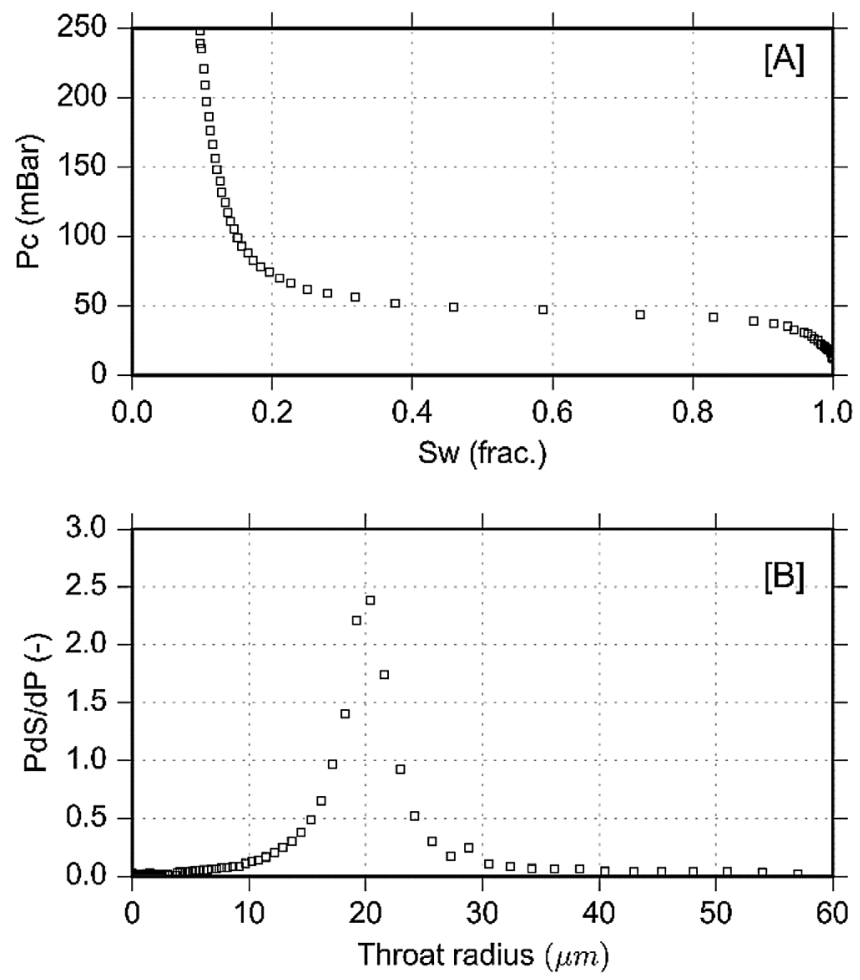

Fig. 3. Mercury intrusion capillary pressure measurement run on a twin Bentheimer sample. (A) Capillary pressure computed for our fluids and rock system, considering an IFT of $45 \mathrm{mN} / \mathrm{m}$ and a contact angle of $0{ }^{\circ} \mathrm{C}$. (B) Throat radius distribution centered around $20 \mu \mathrm{m}$.

contact with the brine has been measured using the Wilhelmy plate method, at ambient temperature. IFT measurements are given in Table 2.

\subsection{Experimental procedure and design}

Height unstable displacements have been conducted in this study, with three parameters varying: the water injection flowrate, the oil viscosity and the core wettability. The experimental corefloods design is summarized in Figure 6, using the water injection flowrate $Q_{\mathrm{inj}}$ and the fluids

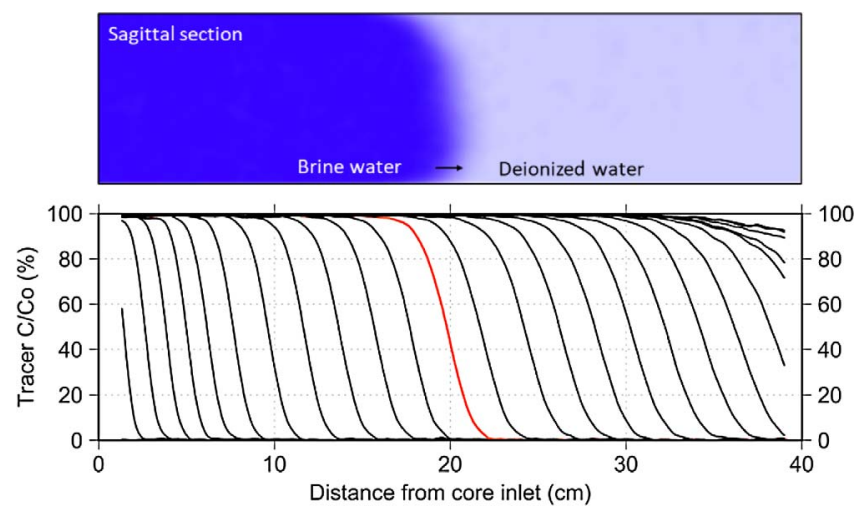

Fig. 4. Tracer concentration profiles monitored during the tracer injection. The profile displayed in red and the sagittal section are monitored at $0.5 \mathrm{PV}$ injected.
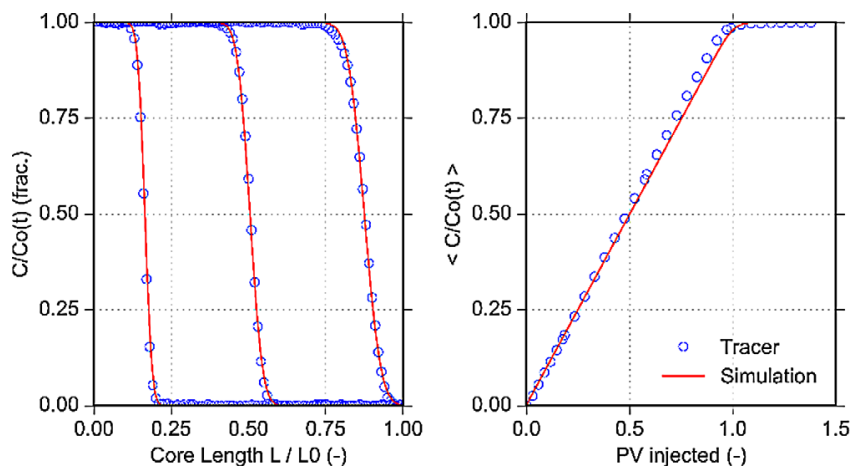

Fig. 5. Core dispersion fitted using a standard analytical solution [27]. The dispersion is fitted using the concentration profile monitored at $0.17 \mathrm{PV}, 0.5 \mathrm{PV}, 0.88 \mathrm{PV}$ injected (left plot) and total tracer concentration (right plot). Dispersion is fitted to $5.4 \times 10^{-9} \mathrm{~m} / \mathrm{s}$, corresponding to a dispersivity of $2 \mathrm{~mm}$. Red lines show the simulated data, blue circles show the experimental data.

viscosity ratio defined as $\mu_{\mathrm{oil}} / \mu_{\mathrm{brine}}$. On this figure, plain markers refer to corefloods conducted on the core with its originate water-wet wettability preserved. Empty markers refer to corefloods conducted on the core after ageing. 


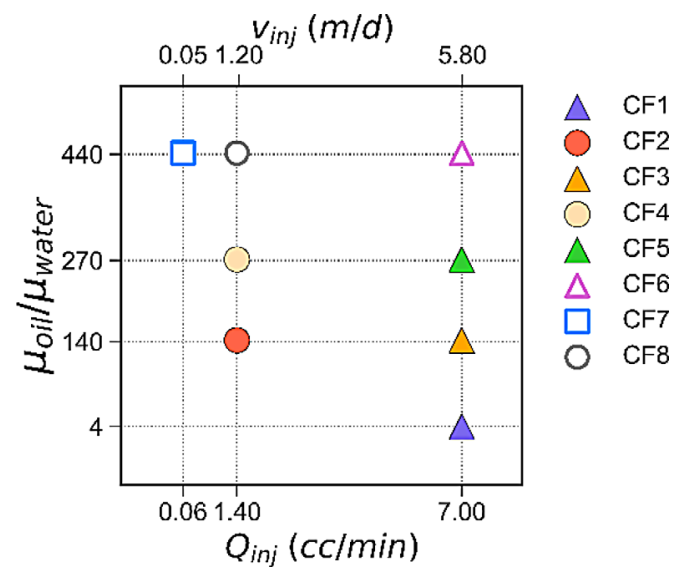

Fig. 6. Experimental corefloods design. Plain markers refer to corefloods conducted before the core ageing (water-wet case). Empty markers refer to corefloods conducted on the aged core (oil-wet). The injection velocity is computed as $v_{\mathrm{inj}}=Q_{\mathrm{inj}} /\left(S^{*} \phi\right)$, with $S$ the core inlet surface.

For the water-wet case (before ageing), five waterflood displacements (named CF1-CF5) have been conducted using varying injection flowrates and oil viscosities. The three mineral oils presented in the previous section have been used: Isopar L $(5 \mathrm{cP})$, Primol $(170 \mathrm{cP})$ and Drakeol $(320 \mathrm{cP})$. Corefloods with the Primol (CF2 and CF3) were conducted first, followed by the coreflood with the Isopar L (CF1). Corefloods with the Drakeol (CF4 and CF5) were conducted at last due its higher viscosity. All water floodings start at $S w i$, reached by injecting the oil at $180 \mathrm{cc} / \mathrm{h}$, until pressure and saturation stabilities are obtained. From $S w i$, the brine is injected for a minimum of 5 pore-volumes. Both the differential pressure and the fluids saturation are monitored during this displacement. After the water flooding, the oil used in the next coreflood is directly injected to reach Swi and replace the oil from the previous experiment. For the coreflood CF1 conduced with lightest oil, Swi is set by flooding with the Primol first, followed by Isopar L injection to replace the Primol. This intermediate step is conducted with the Isopar L oil to have comparable Swi for all the five experiments, considering that its low viscosity could possibly lead to unfavorable displacement.

For the oil-wet case (after ageing the core), three waterflood displacements (named CF6-CF8) are conducted with the DC-O $(540 \mathrm{cP})$ and varying the injection flow only. The same experimental procedure is used to reach $S w i$, with the DC-O injected at $35 \mathrm{cc} / \mathrm{h}$ after each displacement. From Swi, the brine is injected at 7.0, 0.06 and $1.4 \mathrm{cc} / \mathrm{min}$, for CF6, CF7, and CF8 respectively (see Fig. 6).

The core entry face is swept with the brine before proceeding with the injection in the core, using a dedicated outlet port in the inlet face. Homogenous sweeping is ensured by an injection diffuser carved with a double spiral. During the sweeping phase, the flowrate is set to $50 \mathrm{cc} / \mathrm{h}$ for at least $2 \mathrm{~h}$. This step is monitored using the CT-scan for quality-checking. The experimental design of the presented experiments is given in Table 2. Peters \& Flock's number $I_{\mathrm{sc}}$ [8] is computed for each displacement, using equation (1), where $\mu_{\mathrm{o}}$ and $\mu_{\mathrm{w}}$ respectively expressed the oil and brine water viscosity, $v$ is the brine superficial velocity, $K$ is the core absolute permeability, $\sigma_{\text {ow }}$ is the fluids interfacial tension and $C^{*}$ is the dimensionless wettability number, taken to 306 for water-wet core and to 5.45 for the oil-wet core [8]. In their theory, a displacement is unstable when $I_{\mathrm{sc}}>13.56$ which is the case of all performed corefloods but CF1 that was carried out with the lightest oil:

$$
I_{\mathrm{sc}}=\left(\frac{\mu_{\mathrm{o}}}{\mu_{\mathrm{w}}}-1\right) \frac{v \mu_{\mathrm{w}} D^{2}}{C * \sigma_{\mathrm{ow}} K} .
$$

The possibility to have digitations triggered by gravity forces is investigated using the gravity number. The latter is computed for each coreflood using equation (2) [29, 30], where $K$ is the core permeability, $g$ is the gravitational constant, $v$ is the flow velocity, $\mu$ is the displacing fluid viscosity and $\Delta \rho$ is the fluids density contrast. Values are given in Table 2. They suggest that only the coreflood run with the lowest injection flow rate $(\mathrm{CF} 7)$ might be under the influence of gravitational forces $\left(N_{g}>1\right)$. Yet, the gravity number does account for the capillary forces, which can substantially mitigate gravitational digitations, as suggested by Berg and Ott [31] and demonstrated using 3D simulations:

$$
N_{g}=\frac{K g|\Delta \rho|}{v \mu} .
$$

\subsection{Saturation monitoring}

The full core is scanned in $30 \mathrm{~s}$ which allowed to acquire $3 \mathrm{D}$ images with a time interval of 3 min until breakthrough and $20 \mathrm{~min}$ after breakthrough. Voxel size is $0.33 \times 0.33 \times$ $1.25 \mathrm{~mm}$. The lowest resolution $(1.25 \mathrm{~mm})$ is in the core-axis direction. The local saturation, defined at the voxel-scale, is computed from the CT-scans using equation (3). It is computed on a linear scaling between the brine saturated and the oil saturated states (referred to as CT-references). Figure 7 shows a 2D saturation maps extracted from the 3D map, in sagittal and transverse directions. A mean-filter is used with a kernel of $3^{3}$ voxels to reduce noise. All local saturations are displayed using the same color map scale, where hot shades show high oil saturations, and dark shades show high brine saturations (see Fig. 7). Three saturations values are computed from the CT-scans and presented in this work: mean core saturations obtained by averaging all the core local saturations, 1D saturation profiles along the core axis obtained by averaging the transverse cross sections local saturations, and the local saturations computed at the voxel scale. The local saturations are displayed here using a combination of sagittal and transverse slices (see illustration Fig. 7). Positions of the transverse slices are marked by the dotted black lines, at 8,20 , and $32 \mathrm{~cm}$ from the core inlet. In this representation, the water is injected from left to right:

$$
S_{\mathrm{o}}^{i, t}=\left(\mathrm{CT}^{i, t}-\mathrm{CT}_{\text {oil }}^{i}\right) /\left(\mathrm{CT}_{\text {brine }}^{i}-\mathrm{CT}_{\text {oil }}^{i}\right) .
$$

CT-references are acquired for all fluids (brine and mineral oils) except the DC-O (see Fig. 8). They are obtained by 


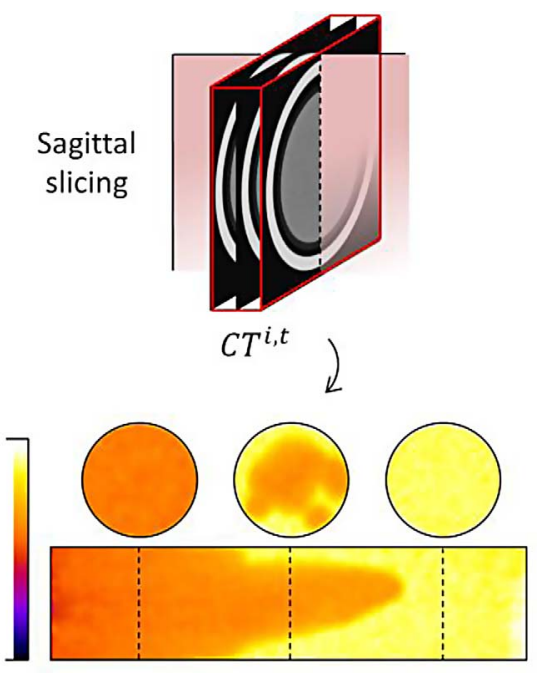

Fig. 7. Illustration of sagittal and transverse slices processing from raw data.

saturating the core with the different fluids through miscible displacement. The mean CT-contrasts between the brine and the different minerals oils are: $157 \mathrm{HU}$ (Hounsfield Unit) with the Isopar L and 135 HU with the Drakeol and Primol. A synthetic reference is computed for the DC-O. It is obtained by using correlations between CT-scan references acquired on different fluids and their CT-contrast measured on the bulk fluid. The average CT-contrast obtained between the brine and the DC-O synthetic reference is $124 \mathrm{HU}$.

Repeatability and stability measurements conducted on the CT-scanner have given a standard error $\delta \mathrm{CT}=1 \mathrm{HU}$. For the brine-water and the mineral-oils CT-references, the standard error is therefore estimated to standard error $1 \mathrm{HU}$. For the DC-O synthetic reference, the standard error is estimated to $2.5 \mathrm{HU}$. The standard error $\delta S o$ for all oil saturation is computed using equations (3) and (4). It is function of the phase saturation. Confidence intervals (IC95, calculated as twice the standard error) are plotted Figure 9 for the 3 mineral oils and the DC-O. IC95 values are higher when the CT-contrast between the oil and the brine is lower. For the mineral oils, the saturation uncertainty is estimated to $\pm 2 \%$. For the DC-O, due to the higher uncertainty for the DC-O reference, the saturation uncertainty is estimated to $\pm 2 \%$ near the brine pole and $\pm 4 \%$ near the DC-O pole. All values used in equation (4) are summarized in Table 3:

\section{See the Equation (4) bottom of the page}

Two additional features used in this work are computed from the CT-scans: the volume swept by the brine $\left(\mathrm{PV}^{\text {swept }}\right)$ and the oil recovery $\left(\mathrm{RF}^{\mathrm{swept}}\right)$, defined at voxelscale, in this swept area behind the water-front. Oil

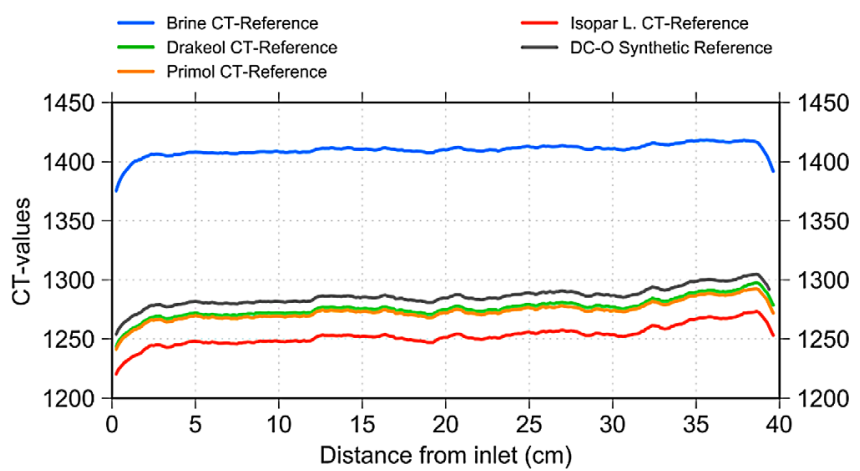

Fig. 8. CT-references of the brine water and the different oils.

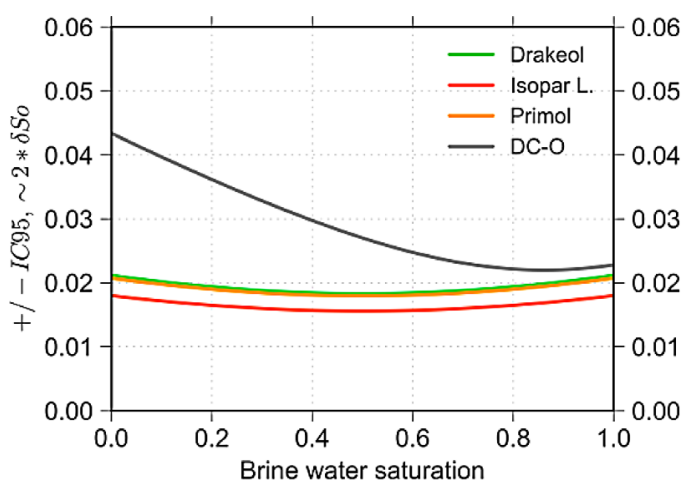

Fig. 9. Saturation confidence intervals (IC95) computed for the different oils. It is computed as twice the standard error $\delta S o . \delta S o$ is computed using equations (3) and (4).

recovery is defined here as the oil production from Swi. These two values have already been defined and computed in previous work by Pavone [32]. They allow to consider the contributions to breakthrough earliness occurring at two different scales: above and below the scanner resolution. Details of the CT-scan processing to compute these values are illustrated in Figure 10. The CT-scan recorded at Swi is subtracted to the CT-scan under consideration (see Fig. 10B). This operation gives the local oil recoveries ( $\mathrm{RF}^{\mathrm{loc}}$ values). The segmentation of the area swept by the brine is performed based on these values. It could have been performed on the oil saturation distribution but the segmentation is less reliable, especially if the local Swi values show a wide distribution. A statistical distribution of oil recoveries is given in Figure 11 to illustrate the segmentation. It shows two populations of values: null (or close to null) for the unswept voxels, and positive values for the swept ones. A threshold of $\mathrm{RF}^{\text {loc }}=7.5$ is applied to separate these two populations. A mask is constructed from this segmentation, delimiting the swept and unswept areas (see Fig. 10C). The PV ${ }^{\text {swept }}$ value is obtained from the voxels count of these two areas. The mask is then applied to

$$
\delta S_{\mathrm{o}}=\sqrt{\left(\frac{1}{\mathrm{CT}_{\text {brine }}-\mathrm{CT}_{\text {oil }}}\right)^{2} * \delta \mathrm{CT}^{2}+\left(\frac{\mathrm{CT}_{\text {oil }}-\mathrm{CT}}{\left(\mathrm{CT}_{\text {brine }}-\mathrm{CT}_{\text {oil }}\right)^{2}}\right)^{2} * \delta \mathrm{CT}_{\text {brine }}^{2}+\left(\frac{\mathrm{CT}-\mathrm{CT}_{\text {brine }}}{\left(\mathrm{CT}_{\text {brine }}-\mathrm{CT}_{\text {oil }}\right)^{2}}\right)^{2} * \delta \mathrm{CT}_{\text {oil }}^{2}} .
$$


Table 3. Average CT-scanner values and standard errors (in HU units) for all fluids, used to compute $\delta S_{\mathrm{o}}$ (see Eq. (4)).

\begin{tabular}{lcccr}
\hline & Isopar L. & Primol & Drakeol & DC-O \\
\hline$<\mathrm{CT}>$ & \multicolumn{5}{c}{ Ranging from $<\mathrm{CT}_{\text {brine }}>$ to $<\mathrm{CT}_{\text {oil }}>$} \\
$<\mathrm{CT}_{\text {oil }}>$ & 1252.7 & 1273.5 & 1276.3 & 1286.0 \\
$<\mathrm{CT}_{\text {brine }}>$ & & & 1410.1 & \\
$\delta \mathrm{CT}$ & 1 & 1 & 1 & 1 \\
$\delta \mathrm{CT}_{\text {oil }}$ & & & 1 & \\
$\delta \mathrm{CT}_{\text {brine }}$ & & & & 2.5 \\
\hline
\end{tabular}
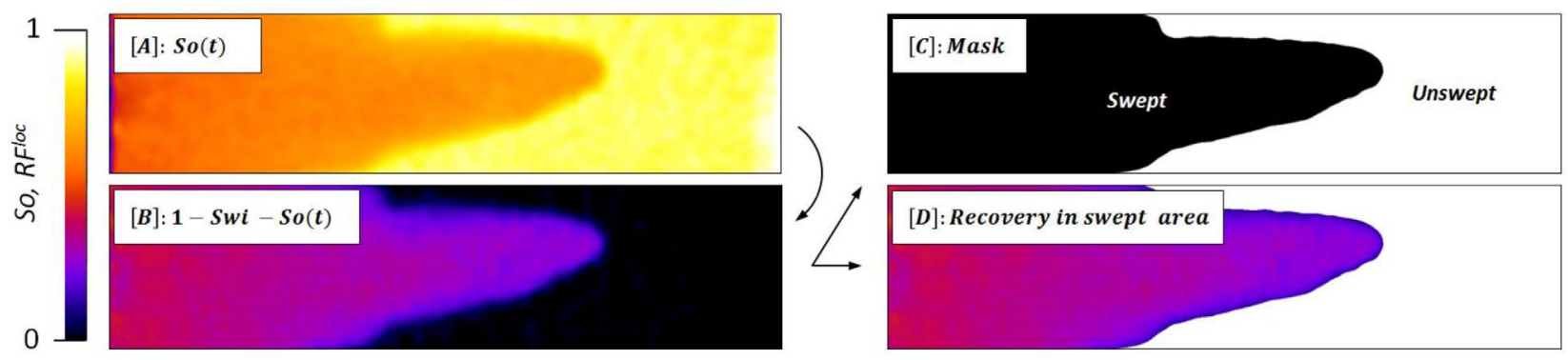

Fig. 10. CT-scan processing to compute the $\mathrm{PV}^{\text {swept }}$ and $R F^{\text {swept }}$ values. (A) Shows the original saturation map, (B) shows the oilrecovery map compute as $1-S w i-S o(t),(\mathrm{C})$ shows the segmentation of the swept area and (D) shows the oil recovery in the swept area selection.

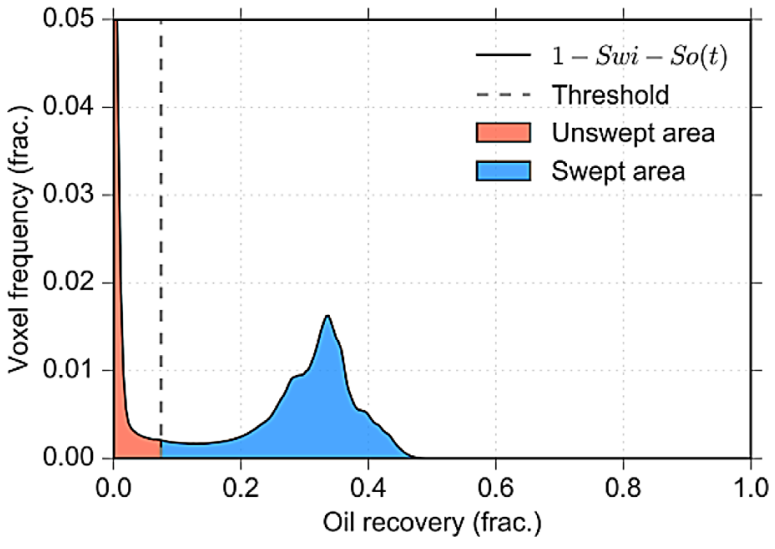

Fig. 11. Local oil recoveries distributions, measured after subtracting the Swi.

measure the $\mathrm{RF}^{\mathrm{swept}}$ value from the average $\mathrm{RF}^{\mathrm{loc}}$ values in the swept areas (see Fig. 10D). Until the water breakthrough occurs, the core oil recovery RF is equal to volume of water injected in the core, when expressed as a number of pore-volumes $\left(\mathrm{PV}_{\mathrm{inj}}\right)$. The two quantities defined above are therefore related until BT as follow (Eq. (5)):

$$
\mathrm{PV}_{\text {inj }}=\mathrm{RF}=\mathrm{PV}^{\mathrm{swept}} * \mathrm{RF}^{\mathrm{swept}} \text {. }
$$

\section{Experimental results}

Experimental data monitored for all corefloods are presented and compared in this section. They are interpreted and discussed in the next section.
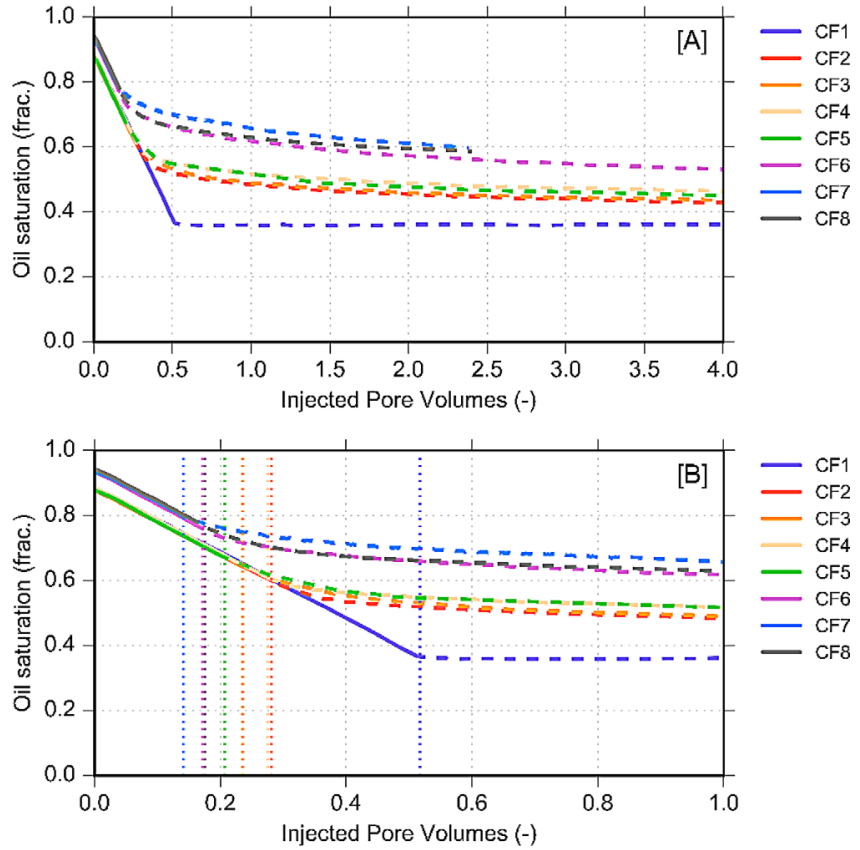

Fig. 12. Oil saturation monitoring of all corefloods (A), zoomed on the first injected pore volume (B). Plain lines show before BT, dashed lines are after BT (separation is indicated by the vertical dotted lines).

\subsection{Saturation monitoring}

The dynamic core saturations measured during the waterflood are compared in Figure 12A for all corefloods. A zoom over the first PV injected is given in Figure 12B. For all 
Table 4. Saturations reached during the corefloods. The oil recovery at breakthrough is computed as $1-S w i-S o(\mathrm{BT})$.

\begin{tabular}{lcccccc}
\hline Coreflood & $\begin{array}{c}\text { Core } \\
\text { treatment }\end{array}$ & $\begin{array}{c}\text { Oil viscosity } \\
(\mathrm{cP})\end{array}$ & $\begin{array}{c}\text { Injection } \\
\text { flowrate }(\mathrm{cc} / \mathrm{min})\end{array}$ & $\begin{array}{c}\text { Swi (frac.) } \\
\text { So at BT } \\
(\text { frac.) }\end{array}$ & $\begin{array}{c}\text { RF at } \\
\text { BT }(\%)\end{array}$ \\
\hline CF1 & No treatment (water-wet) & 5 & 7.0 & 0.117 & 0.381 & 0.503 \\
CF2 & 170 & 1.4 & 0.117 & 0.604 & 0.279 \\
CF3 & & & 7.0 & 0.12 & 0.660 & 0.220 \\
CF4 & & & 1.4 & 0.119 & 0.618 & 0.269 \\
CF5 & & 540 & 7.0 & 0.113 & 0.689 & 0.193 \\
CF6 & Aged core (oil-wet) & & 7.0 & 0.063 & 0.769 & 0.169 \\
CF7 & & & 0.06 & 0.063 & 0.798 & 0.140 \\
CF8 & & & 1.4 & 0.053 & 0.776 & 0.171 \\
\hline
\end{tabular}

figures, time is expressed as the number of Pore-Volume $(\mathrm{PV})$ injected in the core. This scaling is chosen to be able to compare the corefloods despite the different injection flowrates.

The first element to be compared is the Swi values reached at the end of the drainage. They are comparable for corefloods of same wettability system (see values in Tab. 4). For the initial water-wet system, Swi values range from $11.3 \%$ to $12 \%$. For the altered oil-wet wettability, the Swi are lower, ranging from $5.3 \%$ to $6.3 \%$. The lower Swi values measured after ageing the core is consistent with Craig's rule of thumb [33]. For these corefloods (CF6CF8), the DC-O was used to reached Swi, which has the highest viscosity. It may have also contributed to reach lower Swi values. The comparison of the saturation curves (Fig. 12) shows differences for all corefloods. This result was expected since different flowrates, oil viscosities or core wettability are used here. Three mains groups can be identified when looking at the oil saturation reached after $1 \mathrm{PV}$ injected: (i) coreflood $\mathrm{CF} 1$ alone, characterized by a low oil saturation $(<0.4)$; (ii) corefloods CF2-CF5 characterized by intermediate oil saturations $(\sim 0.5)$; and (iii) corefloods CF6-CF8 characterized by high oil saturation $(>0.6)$. These groups point-out again a dominant effect of the wettability on the displacement. The coreflood CF1 was expected to show a different behavior as only this coreflood was predicted to be stable (see $I_{\mathrm{sc}}$ values in Tab. 2).

As mentioned above, the easiest method to characterize unstable displacement is to measure the breakthrough earliness (referred to as RF at BT). The latter is expressed in number of pore-volume injected in the core until BT. In Figure 12, the dotted sections of the curves show the saturation monitored after BT. A standard method to estimate the BT from the saturation curve it to detect the slope break: the decrease of saturation is linear to the time until BT, the linearity is lost after BT. However, a clear break in the slope is not always observable, especially when the displacement is unstable. Here, the BT is estimated using the saturation profiles (see Fig. 13) and the local saturations when needed (Figs. 14 and 15). The oil recoveries measured at BT are summarized for all corefloods in Table 4. Values range from 0.14 for the coreflood CF7 (earliest BT) to 0.503 for the coreflood CF1 (latest BT). The three groups of corefloods identified above are no longer observable. It underlines the complexity to characterize unstable displacements: the comparison we get may be different when a different descriptor is used. Hence the need to be the most exhaustive in the description of these floodings. The coreflood CF1 is still demonstrating a singular behavior, while the other corefloods show graduated differences. The RF at $\mathrm{BT}$ are overall consistent with the $I_{\mathrm{sc}}$ number computed in Table 2. The effect of the oil viscosity can be seen for the water-wet case only, with the two sets of corefloods CF1-CF3-CF5 ( $\left.Q_{\text {inj }}=7 \mathrm{cc} / \mathrm{min}\right)$ and CF2-CF4 $\left(Q_{\text {inj }}=1.4 \mathrm{cc} / \mathrm{min}\right)$. Corefloods conducted with the lightest oil show the latest $\mathrm{BT}$ while the ones conducted with the heaviest oils show the earliest BT. For the water-wet corefloods, the effect of the injection flowrate is visible when comparing CF2 with CF3, and CF4 with CF5 (Tab. 4). Results suggest an earliest BT when the flowrate is increased. These observations are in agreement with most recent studies conducted with this wettability system $[20,34]$. This trend appears to be no longer valid after ageing the core. The corefloods $\mathrm{CF} 6$ and $\mathrm{CF} 8$ have comparable oil recovery at BT while having different injection flowrate. The latter is increased by more than two decades between CF6 and CF8, but only a limited impact is observed of the BT earliness. This opposite or limited effect of the injection flow on the BT rate for the oil-wet case has been recently observed by [13].

The saturation curve monitored for the coreflood CF1 shows a specific production behavior, where no additional oil is produced after BT. This production dynamic usually signs for a piston-like displacement. The saturation profiles (see Fig. 13) clearly confirm this type of displacement for this coreflood. They demonstrate a sharp transition of saturation ahead and behind the waterfront. No front digitation is observed at macro-scale (above the voxel size), the core sweeping is stable and efficient. A perfect stability was not expected since the viscosity ratio $\left(\mu_{\text {isopar }} / \mu_{\text {brine }}=4\right)$ is still unfavorable. However, it explains the latest BT observed for CF1. Inversely, the BT earliness measured for all other corefloods is caused by non-piston-like displacements. The saturation profiles (Fig. 13) show a water-front spreading over several centimeters. Oil is still produced after breakthrough by the time the rest of the waterfront reaches the core outlet. The profiles monitored for the coreflood 

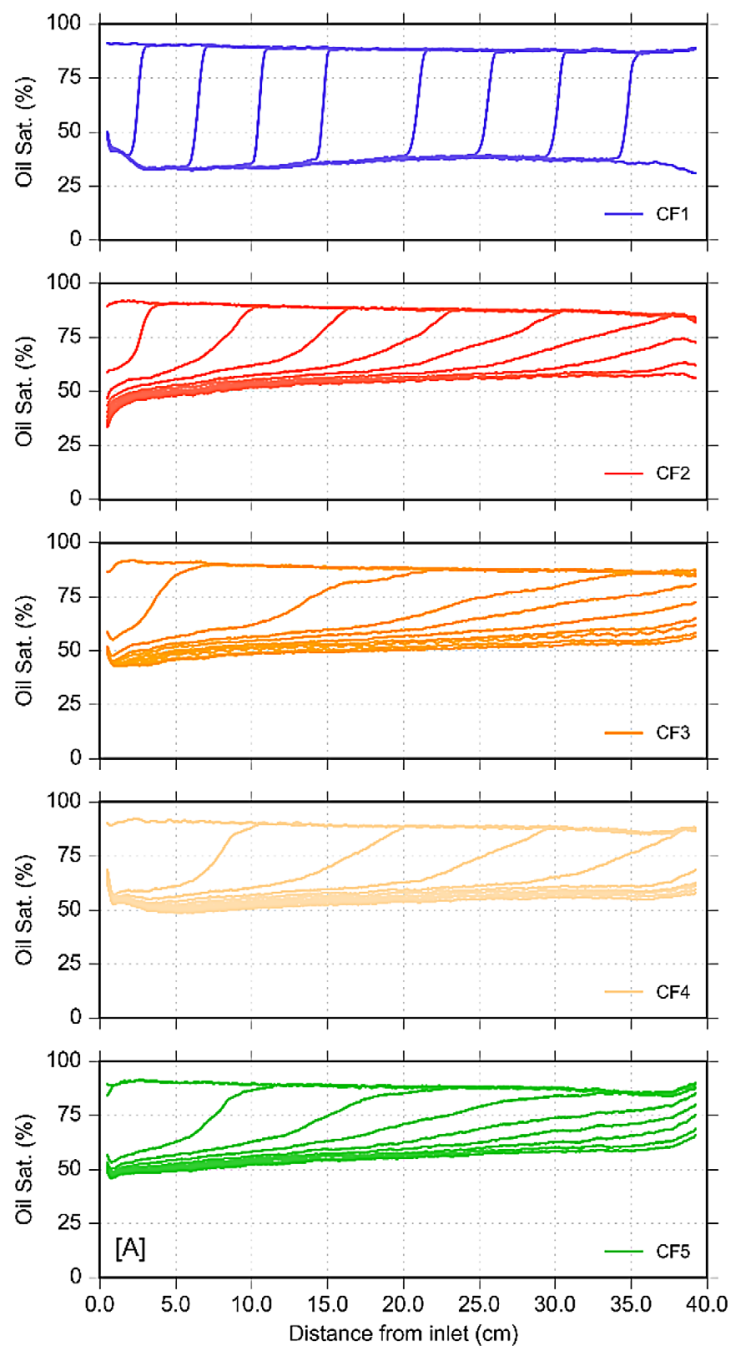
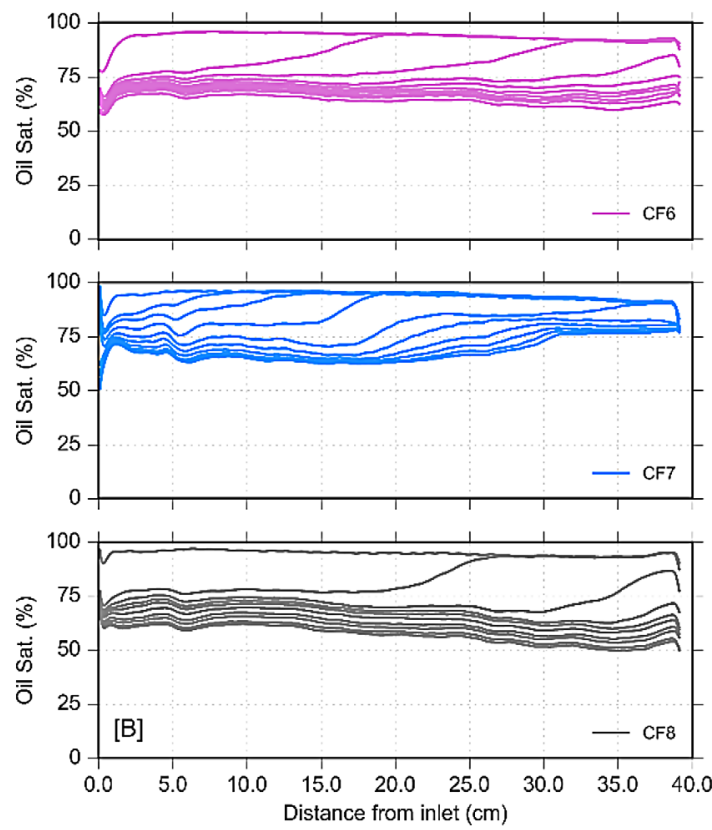

Fig. 13. Oil saturation profiles along the core axis for all corefloods, for the water-wet case (A) and the oil-wet case (B).

conducted with the lowest injection flowrate (CF7) demonstrate an atypical behavior with at least two waterfronts observed during the flooding. The local saturations given in Figures 14 and 15 show the front digitations causing the non-piston-like displacements. For the water-wet case (Fig. 14), these digitations take the form of large fingers, globally growing at the center of the core. When multiple fingers are initially formed (for CF3, CF4, and CF5), they slowly merge during the injection. The front digitations take a completely different shape after ageing the core (Fig. 15). Large fingers are not observed however the water-front exhibits a higher complexity, with viscous fingers formed at a smaller scale. The flooding pattern monitored for the coreflood CF7 clearly shows a change in the flooding dynamic with a preferential invasion of the lower part the core. This change of invasion explains the atypical saturation profiles observed earlier. The shape of the front digitations observed for the two wettability systems considered here is in good agreement with results obtained by de Haan [15].

The local saturation monitoring allows us to compare the oil recovery behind the waterfront. As well as an unstable water-front, the oil-recovery is a contribution to BT earliness. The previous three groups of corefloods can be identified again. The coreflood CF1 shows the lowest oil saturation, around 0.37 . The other water-wet corefloods (CF2-CF5) all demonstrate a comparable residual oil saturation measured around 0.6. Finally, the oil-wet corefloods show the highest oil saturations. The local saturations, given at two different time steps (at BT and half the BT), suggest a reduction of the oil saturation behind the front during the flooding, which was not observed for all other corefloods until BT. For CF6-CF8, the oil saturation behind the front is measured around $82 \%$ at halt BT, and reduced by $5 \%$ at BT. These observations suggest again the main influence of the core wettability on the water flooding.

\subsection{Differential pressure monitoring}

The Differential Pressures $(\mathrm{dP})$ recorded during these displacement are showed in Figure 16A for the water-wet cases (CF1-CF5) and in Figure 16B for the oil-wet cases (CF6$\mathrm{CF} 8$ ). For all corefloods except $\mathrm{CF} 1$, dP monitoring shows a decrease of the differential pressure during all the water 

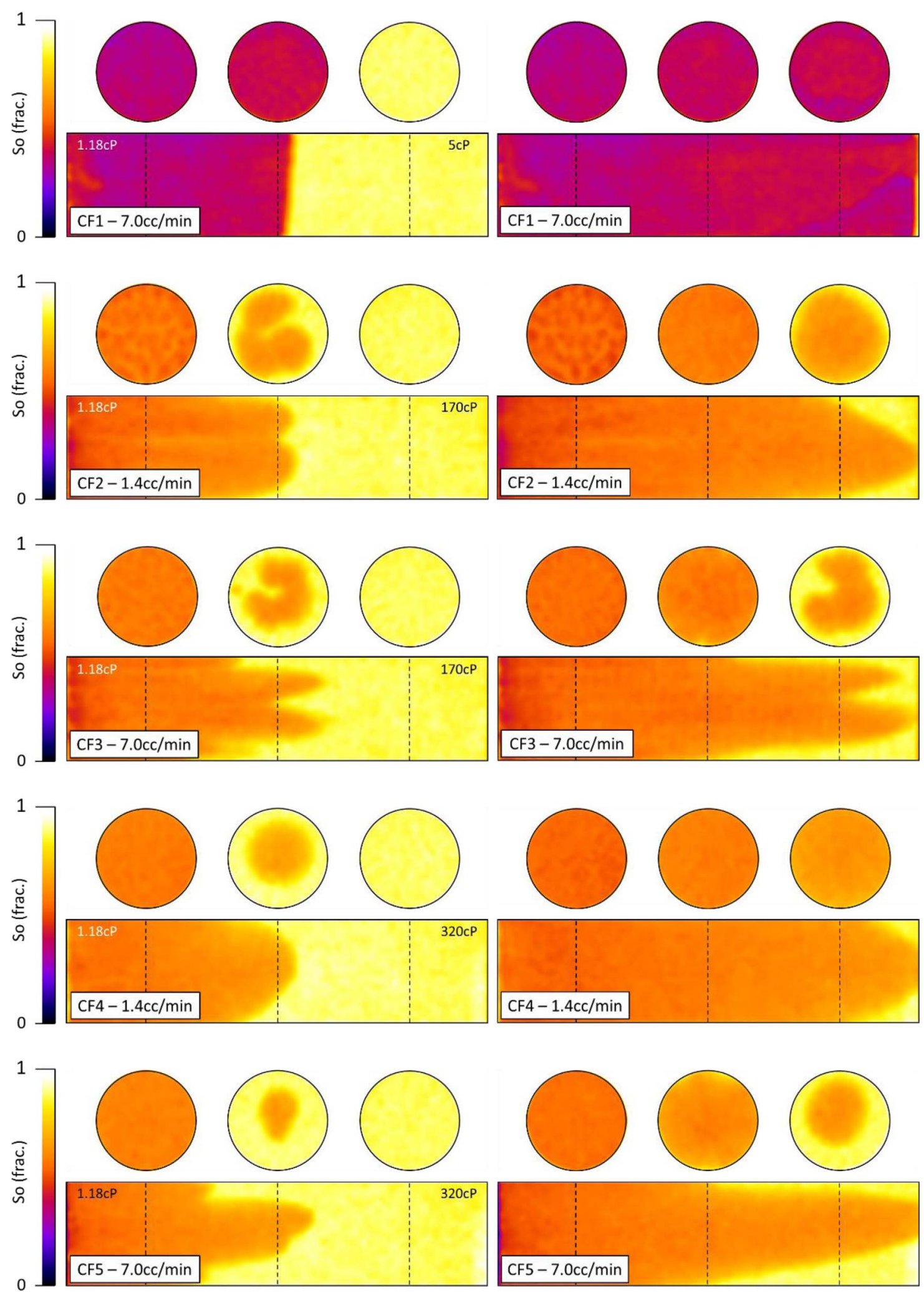

Fig. 14. 2D visualization of the front digitations using sagittal and transverse slices, for the water-wet case before the core ageing (coreflood CF1-CF5). The displacement is displayed at half the breakthrough on the left and at breakthrough on the right. Positions of the transverse slices are marked by the dotted black lines. The sagittal slicing is illustrated in Figure 7. Injected and displaced fluid's viscosities are given respectively on the top left and top right of the sagittal slicing measured at half the breakthrough. 

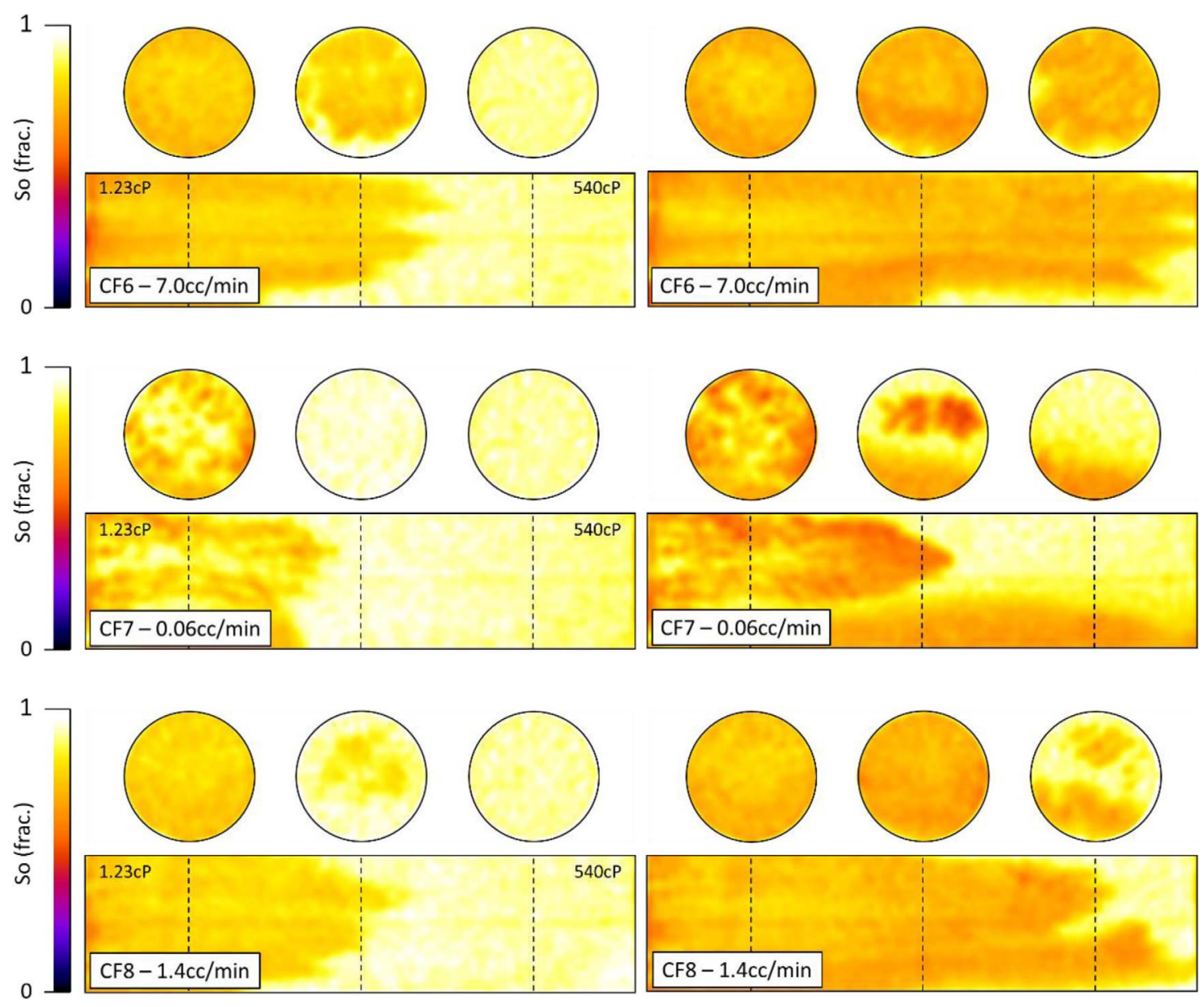

Fig. 15. 2D visualization of the front digitations using sagittal and transverse slices, for the oil-wet case after the core ageing (coreflood CF6-CF8). The displacement is displayed at half the breakthrough on the left and at breakthrough on the right. Positions of the transverse slices are marked by the dotted black lines. The sagittal slicing is illustrated Figure 7.

injection. This is easily explained by the fluid's viscosities. The replacement of a viscous fluid by a fluid of lower viscosity causes the reduction of the pressure losses in the porous media. The opposite trend is observed for the coreflood CF1 conducted with the lightest oil (Isopar L), with the $\mathrm{dP}$ increasing during the water flooding. The previous observations with the local saturations have demonstrated a perfect piston-like displacement. In this type of displacement, only oil is flowing head of the waterfront at $S w i$, and only brine is flowing behind the waterfront at Sorw. The pressure drop from inlet to outlet is driven by mainly two values: the oil relative permeability at Swi and the water relative permeability at Sorw, respectively referred to as $k_{\text {rom }}$ and $k_{\text {rwm }}$. An increasing $\mathrm{dP}$ during the flooding simply requires having a relative permeabilities contrast that overrides the unfavorable viscosity ratio: $k_{\mathrm{rom}} / k_{\mathrm{rwm}}>\mu_{\mathrm{o}} / \mu_{\mathrm{w}}=4$. This condition is easily met for strongly water-wet cores $[33,35]$. The piston-like displacement allows to use a $1 \mathrm{D}$ two-phases flow simulator to simulate this coreflood. Results of the simulation are displayed in Figure 17. Experimental data (oil saturation and $\mathrm{dP}$ ) are fitted using $k_{\mathrm{rom}} / k_{\mathrm{rwm}}=6.6$. As suggested by most studies $[1,4,5,12]$, the fluids contrast alone is not a relevant indicator to predict the front stability. This indicator doesn't provide a good characterization of the fluids mobilities when flowing in a porous structure that exhibit a strong preferential affinity (i.e. the core wettability) for one of the fluids. For the coreflood CF1, the fluids mobility ratio (using the end-points definition $\left.M=\left(\mu_{\mathrm{o}} * k_{\mathrm{rwm}}\right) /\left(\mu_{\mathrm{w}} * k_{\mathrm{rom}}\right)\right)$ is estimated to $M=0.6$. This value is consistent with the lack of viscous digitations observed for this coreflood.

\section{Discussion}

So far, the digitations have been quantified only using the breakthrough earliness. At least two different contributions to early BT have been identified: (i) macro-scale digitations and (ii) the oil recovery behind the waterfront. The previous observations have suggested that BT was impacted by the injection flowrate, the oil viscosities, and the core wettability. This section aimed to discuss their effects using the previous observations. 

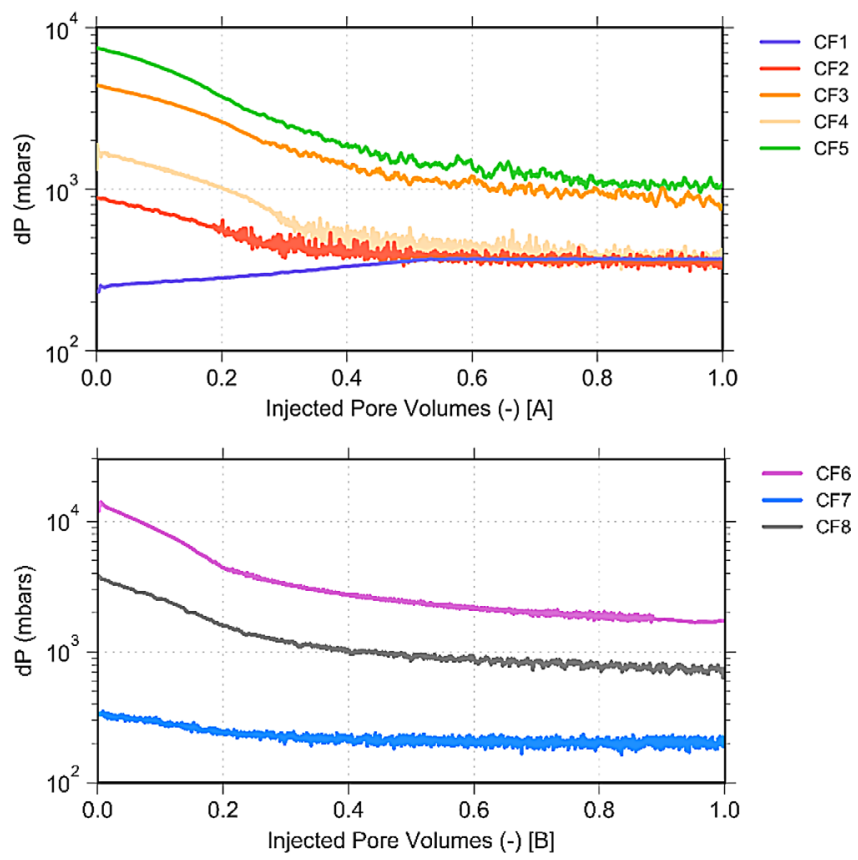

Fig. 16. Differential Pressure $(\mathrm{dP})$ recorded during the corefloods, for the water-wet case (A) and the oil-wet case (B).
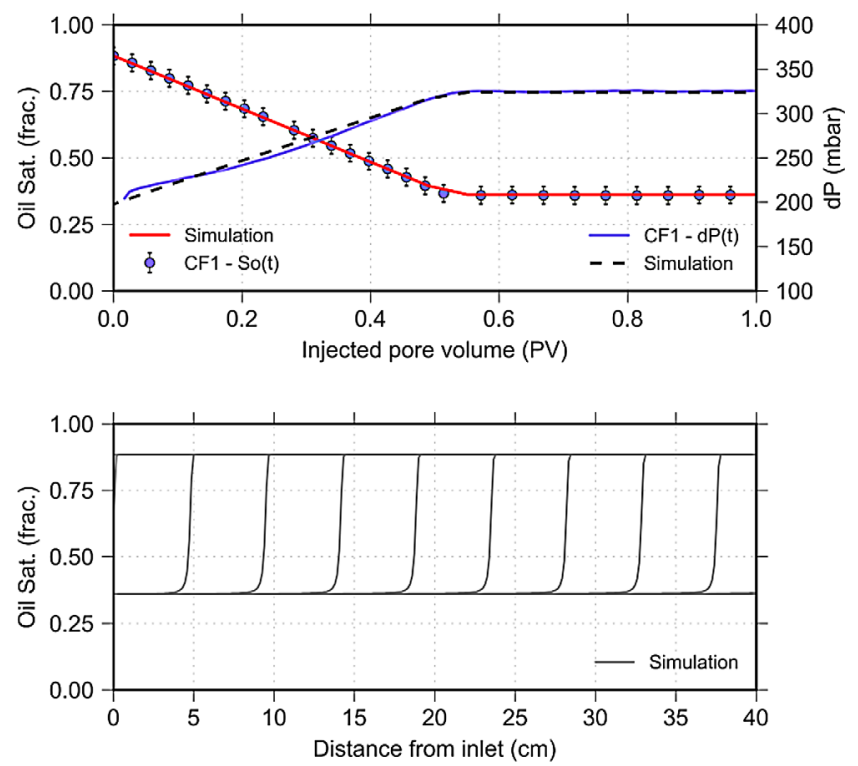

Fig. 17. Simulation of coreflood CF1 using CYDAR, with $k_{\text {rom }}$ and $k_{\text {rwm }}$ taken to 0.6 and 0.9 respectively.

\subsection{Water breakthrough versus dimensional numbers}

The oil recovery measured at $\mathrm{BT}(\mathrm{RF}$ at $\mathrm{BT})$ is plotted in Figure 18 as a function of the $N_{I}$ number (Tab. 5) defined by Doorwar in his thesis [11] (see Eq. (6)). This dimensionless number demonstrated good results to scale BT earliness for many corefloods conducted with varying experimental conditions. In regard of the experimental design used here (see Fig. 6), the $N_{I}$ number can be simplified as described in equation (6), to consider only the varying parameters:

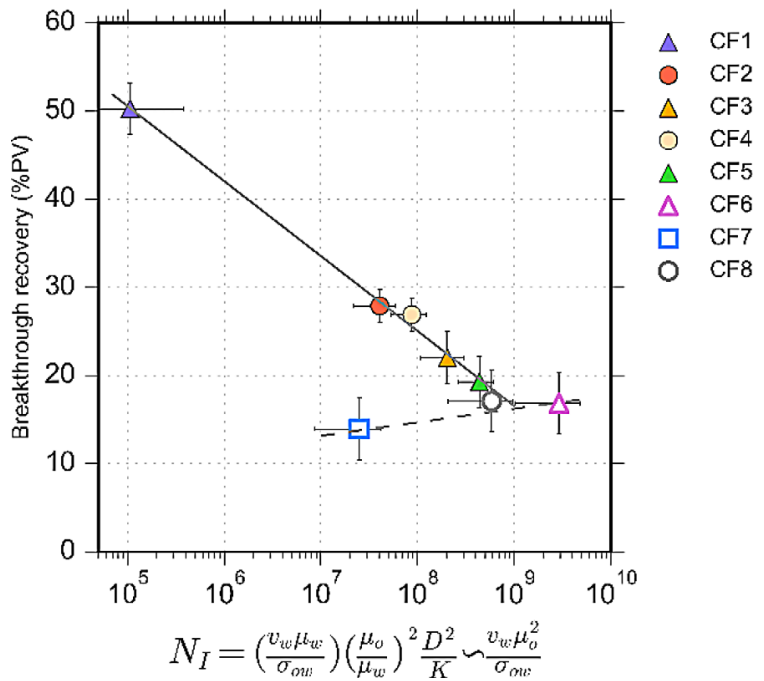

Fig. 18. Oil recovery at breakthrough ( $\mathrm{RF}$ at $\mathrm{BT})$ versus Doorwar's number $N_{I}$. The abscissa error bars account for the fluid viscosities, the IFT and the injection flow-rate uncertainties. The ordinate error bars account for the saturation confidence intervals and the temporal resolution of the CTmonitoring. Square, circle and triangle markers respectively refer to corefloods conducted at $0.06,1.4$ and $7.0 \mathrm{cc} / \mathrm{min}$. Plain markers refer to corefloods conducted before the core ageing (water-wet case). Empty markers refer to corefloods conducted on the aged core (oil-wet).

Table 5. Oil recovery at breakthrough (RF at BT) and Doorwar's number $N_{I}$.

\begin{tabular}{lcc}
\hline Coreflood & RF at BT $(\%)$ & $N_{I}(-)$ \\
\hline CF1 & 0.503 & $1.1 \mathrm{E}+05$ \\
CF2 & 0.279 & $4.1 \mathrm{E}+07$ \\
CF3 & 0.220 & $2.0 \mathrm{E}+08$ \\
CF4 & 0.269 & $8.7 \mathrm{E}+07$ \\
CF5 & 0.193 & $4.4 \mathrm{E}+08$ \\
CF6 & 0.169 & $2.9 \mathrm{E}+09$ \\
CF7 & 0.140 & $2.5 \mathrm{E}+07$ \\
CF8 & 0.171 & $5.9 \mathrm{E}+08$ \\
\hline
\end{tabular}

$$
N_{I}=\frac{v_{\mathrm{w}} \mu_{\mathrm{w}}}{\sigma_{\mathrm{ow}}}\left(\frac{\mu_{\mathrm{o}}}{\mu_{\mathrm{w}}}\right)^{2} \frac{D^{2}}{K} \sim \frac{v_{\mathrm{w}} \mu_{\mathrm{o}}^{2}}{\sigma_{\mathrm{ow}}} .
$$

Figure 18 clearly shows the lack of scaling between corefloods of different wettability systems (plain markers for water-wet, empty markers for altered oil-wet). This was expected as the core wettability is not taken into account in this $N_{I}$ number, while the previous observations demonstrated its impact on the digitation behavior. This limit of the $N_{I}$ number was already pointed out by [13]. For the water-wet case (corefloods CF1-CF5), the oil-recoveries at breakthrough show a possible correlation with Doorwar's number. It is fitted using a logarithmic regression. The size of the data set $(5 \mathrm{CF})$ and the number of varying 
parameters (3 parameters) don't allow validation of the consistency of this model (or any model). Logarithmic trends can be easily mistaken with power-law trend [36]. Only the general trends can be interpreted here. Mainly two trends can be seen on Figure 18:

(i) For the water-case, an earlier BT is observed when higher oil viscosity or injection flowrate is considered. This trend is no longer observed after altering the core to oil-wet (or less water-wet).

(ii) Earlier BT is observed after ageing the core.

The effect of the fluids viscosities is easily understandable as it directly impacts the fluids mobility ratio and therefore the water-front stability. The previous analysis on the differential pressure monitoring demonstrated the limitation of only using the fluids viscosity to predict the onset on digitations. The coreflood CF1 has shown a situation where an unfavorable mobility ratio was overridden by favorable relative permeabilities curves ( $k r$-curves), resulting in a favorable fluids mobility and a stable displacement. This result would probably not have been observed for this viscosity ratio after ageing the core, as the $k r$-curves are no longer favorable for the oil-phase [33, 35]. A possible improvement to scale the digitation behavior for different wettability systems could be to use a mobility ratio that integrates the fluids relatives permeabilities. For all other corefloods, the viscosity ratio is too unfavorable to be overridden by $k r$-curves, resulting in the formation of viscous digitations (Figs. 14 and 15). It is important to remind that the Bentheimer core used here is overall homogeneous and is therefore not contributing to the propagation of the digitations [28]. However, small scale heterogeneities may have triggered them. The same goes for possible imperfect injection boundary conditions, despite the efforts made to mitigate them.

The local saturation monitoring and the saturation profiles show how the digitations grow during the injection. Their shapes for different time-steps are globally selfsimilar, when normalized by the front advancement. For the water-wet case, the merge of the main fingers was observed. This behavior was already observed by Brock and Orr [28]. They explained their observations using a viscous crossflow mechanism. The low viscosity brine digitations carry high pressures in the viscous oil, leading to a transverse pressure gradient. The finger's transverse spread reduces the BT earliness by invading by-passed areas. For a strongly water-wet core, the capillary pressure may act similarly by causing the fingers to imbibe in all directions. This observation has been demonstrated with 2D numerical simulations [31]. For an oil-wet core, this crossflow is partially dampened by the capillary pressure, limiting the finger's transverse growth, thus favoring the axial growth and the BT earliness. This mechanism may explain the massive and cohesive digitation's shape for the water-wet case while looking more fragmented after ageing the core. The digitations observed for the coreflood CF7 show a unique trend compared to all other corefloods. Their shapes are clearly not self-similar for different time-steps. The preferential invasion of the lower part of the core, most likely driven by gravity forces, mitigates the growth of the initial digitations. The gravity number computed for this coreflood (see Tab. 2) suggested this could happen.

The different digitations patterns observed for the two wettability systems were also justified by de Haan [15] using an idealized pore-scale approach. For the water-wet case, the capillary pressure is causing the brine to move faster in restrictions (throats) than in larger cavities (pores). In this situation, oil is left trapped as disconnected ganglia behind the water-front as it goes forward. The reverse preferential invasion is observed for the oil-wet case, leaving the oil phase partially connected though the restrictions filled with and grains coating. It leads to a less coherent frontinterface for the oil-wet system, resulting in the formation of a larger number of independent fingers. Accordingly to this mechanism of formation, fingers are expected to be developed at a lower scale for the oil-wet case. Our observations for the oil-wet case (CF6-CF7) may not support these predictions as small-scale fingers are only observed during the early stages of the coreflood CF7. A possible explanation could be that these digitations occur below the CTscanner resolution. A second consequence of the different trapping mechanisms for the two wettability systems considered here is the oil recovery in the swept areas. The local saturation demonstrated a lower oil-recovery in the swept areas for the oil-wet case. The relation between oil-recovery and core wettability is not straightforward [37], however, it is obviously that a lower oil-recovery will contribute to an earlier BT. The microscopic oil recovery, measured at the voxel scale, may possibly account for some viscous fingering occurring at a sub-voxel resolution, explaining the lower values measured for the corefloods.

The effect of the injection flowrate on the BT earliness for the two wettability systems can be discussed now that the effect of the wettability has been considered. As seen previously, capillary forces contribute to stabilize the waterfront by dampening the axial growth for the water-wet case only. When the water injection is increased, the viscous forces are increased, and the duration of injection is reduced. Both these effects mitigate the stabilization of the waterfront by spontaneous imbibition. For an oil-wet core (or a less water-wet core), the spontaneous imbibition is suppressed (or reduced). As follow, the effect of the injection flowrate is mitigated.

All of the oil viscosity, the injection flowrate and the core wettability have demonstrated that they impact the digitation behavior. However, observations have suggested that their effects couldn't be considered individually. The effect of the injection flowrate on digitations is related to the core wettability, as seen previously. A study by Tang and Kovscek [34] showed that the effect of the injection flow is also related to the fluids mobility ratio: they observed no effect of the injection flowrate when favorable fluids mobility ratios are considered. The core wettability has a dual contribution here. It strongly affects the digitations pattern and changes the local oil-recovery. The first contribution of the wettability and the injection flow have a mitigated effect on the digitations if they are not initially triggered by viscous forces, described by the fluid's mobility ratio. The interplay of the different flowing conditions mitigates 
the possibility of constructing a unique scaling dimensionless number.

\subsection{Dynamic characterization of the digitations}

The local saturation monitoring for the oil-wet case has suggested the oil recovery in the swept area was improving during the sweeping. It suggests the limit of quantifying the digitations for a unique time-step. Additionally, the local saturation monitoring has shown all the complexity and diversity of the digitation pattern, especially when comparing the oil-wet and the water-wet cases. As mentioned in the saturation monitoring section, the oil recovery measured for all time-steps can be split in two values: the volume fraction swept by the brine $\mathrm{PV}^{\text {swept }}$ and the oil-recovery behind the water-front $\mathrm{RF}^{\mathrm{swept}}$ defined at the voxel scale. These two values are related to the oil recovery through equation (5). The definition of these two contributions is similar to a previous study by Pavone [32]. Lenormand et al. [10] interpreted them with two different fingering mechanisms: viscous fingering occurring at the macro-scale and capillary fingering occurring at the pore-scale. As the scanner resolution falls between these two scales, the $\mathrm{PV}^{\text {swept }}$ and $\mathrm{RF}^{\text {swept }}$ values computed here can be read to quantify the viscous fingering and the capillary fingering contributions to early BT.

The computation of these two values provides useful information to better characterize the digitations and understand the BT earliness. It shows how the oil is left in the core after the water flooding: mostly trapped by capillary forces as disconnected ganglia or by-passed at a larger scale due to unfavorable fluid mobility ratio. An illustration is given in Figure 19 for a coreflood for which an oil recovery of 0.3 would be measured at BT. The blue line draws all the possible combinations of $\mathrm{PV}^{\text {swept }}$ and $\mathrm{RF}^{\text {swept }}$ values (see Eq. (5)). It ranges from two opposite situations:

- $\mathrm{PV}^{\text {swept }}=100 \%$ with low $\mathrm{RF}^{\mathrm{swept}}$ values: the core is perfectly swept (piston-like), the BT earliness is only caused by capillary fingering (upper part of the graph, see illustration $\mathrm{A}$ ).

- $\mathrm{RF}^{\text {swept }}=100 \%$ with low $\mathrm{PV}^{\text {swept }}$ values: there is no capillary fingering, the BT earliness is only caused by viscous fingering (right part of the graph, see illustration B).

All possible combinations exist between these two extreme scenarios (see illustration C). This plot allows an easy comparison and identification of the fingering mechanisms prevailing for each coreflood configuration. The values measured at BT for all corefloods are given in Table 6 and plotted in Figure 20A. The plain lines show all the possible combinations of $\mathrm{PV}^{\text {swept }}$ and $\mathrm{Mic}^{\text {rec }}$ values for a given BT earliness (see illustration Fig. 19). Globally, all corefloods fall in the upper part of the graph. The lowest $\mathrm{PV}^{\text {swept }}$ value is measured for CF7 to $65 \%$. It suggests that the viscous fingering is not the main mechanism contributing to early BT. This was already visible on the local saturation monitoring: the sagittal and cross sections recorded at BT shew the core was overall well swept, despite the clear viscous digitations. The coreflood $\mathrm{CF} 1$ that showed

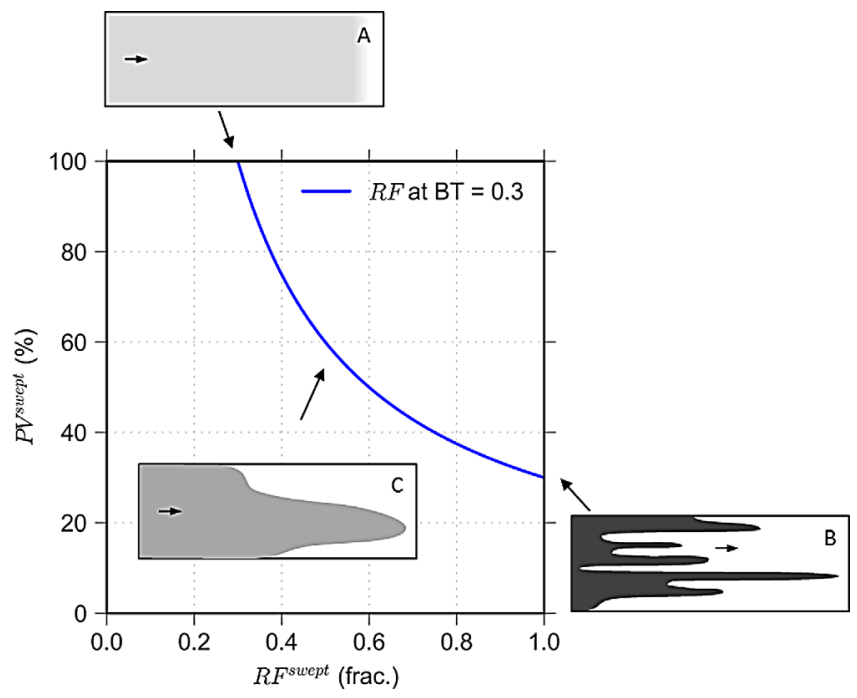

Fig. 19. Illustration of the digitation's characterization using the $\mathrm{PV}^{\text {swept }}$ and $\mathrm{RF}^{\text {swept }}$ values. Three possible digitations patterns $\mathrm{A}, \mathrm{B}$ and $\mathrm{C}$ are shown resulting in the same RF at BT of 0.3. In this illustration, darker shades suggest higher watersaturation.

the piston-like displacement falls in the first extreme scenario described above, with the BT earliness explained by capillary fingering only. All corefloods of same wettability (except CF1) show comparable $\mathrm{RF}^{\mathrm{swept}}$ values: around 0.3 before ageing and around 0.2 after ageing. For these sets of corefloods (CF2-CF5 and CF6-CF8), the different BT earliness is mostly explained by different $\mathrm{PV}^{\text {swept }}$ values. The number of experiments conducted here is not sufficient to correlate the $\mathrm{PV}^{\text {swept }}$ and $\mathrm{RF}^{\text {swept }}$ values with the experimental design presented in Figure 6. A comparable analysis has been conducted by Pavone [32]. He demonstrated that these two contributions could be correlated to two different scaling groups. Still, this comparison shows that the earlier BT measured for all the oil-wet corefloods are explained by a lower oil recovery behind the waterfront, not by more viscous digitations. This result was not expected considering the previous observations. The lack of the front stabilization by capillary forces was expected to lead to more viscous fingering for the oil-wet corefloods. As mentioned above, the voxel size must be kept in mind when interpreting this result as sub-voxel viscous fingering may occur.

The $\mathrm{PV}^{\text {swept }}$ and $\mathrm{RF}^{\mathrm{swept}}$ values measured for all timesteps are plotted in Figure 21, and cross-plotted in Figure 20B. Measurements before and after BT are displayed with plain and dashed lines respectively. They provide meaningful information regarding the production mechanisms during the different stages of the flooding. An additional property is given in Figure 22: the waterfront advancement as function of the number injected $\mathrm{PV}$. It is defined as the further distance reached by the digitations, for each time-step. For the water-wet case, the front advancement is globally linear to the volume injected, suggesting a stability in the digitation's growth. The advancement speed is however slightly decreasing for the oil-wet coreflood, suggesting an improvement of the 
Table 6. $\mathrm{PV}^{\text {swept }}$ and $\mathrm{Mic}^{\text {rec }}$ values measured at BT on the $3 \mathrm{D}$ CT-scans.

\begin{tabular}{lcccccccc}
\hline & CF1 & CF2 & CF3 & CF4 & CF5 & CF6 & CF7 & CF8 \\
\hline RF $^{\text {swept }}$ (frac.) & 0.513 & 0.317 & 0.303 & 0.299 & 0.288 & 0.195 & 0.215 & 0.208 \\
PV $^{\text {swept }}$ (\%) & 98 & 88 & 73 & 90 & 67 & 86 & 65 & 82 \\
\hline
\end{tabular}
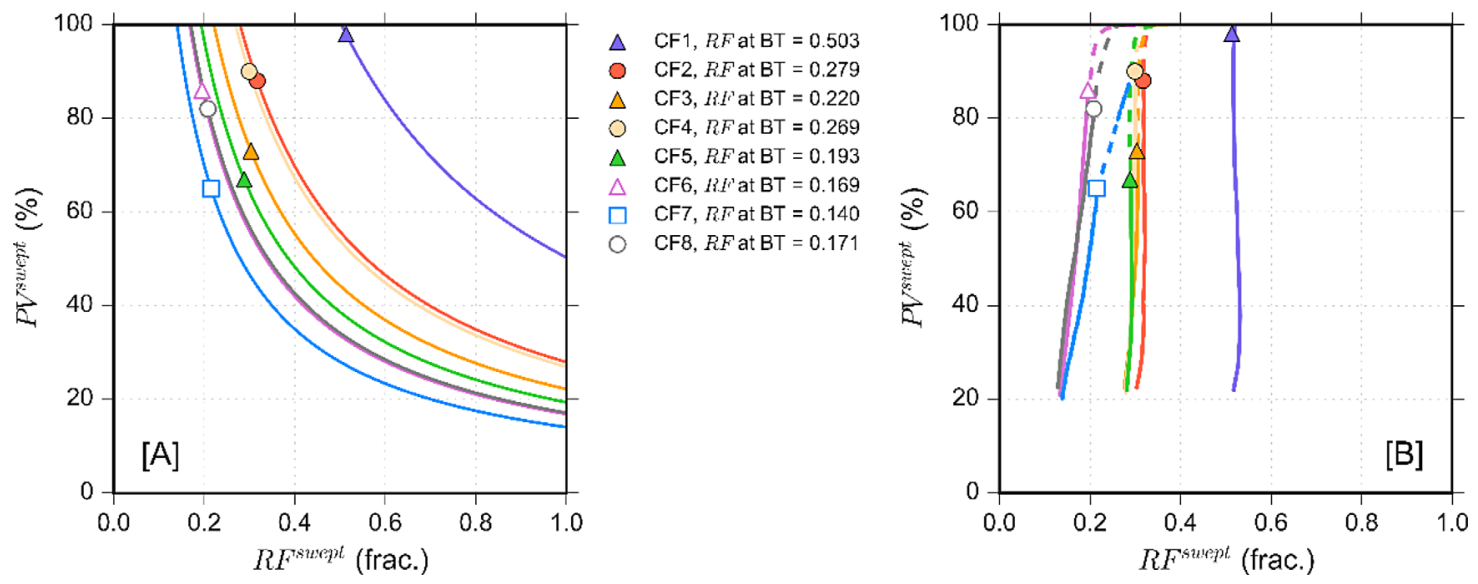

Fig. 20. (A) $\mathrm{PV}^{\text {swept }}$ and $\mathrm{RF}^{\text {swept }}$ values measured at BT. The lines show all possible combination of values $\left(\mathrm{PV}^{\text {swept }}\right.$ and Mic $\left.{ }^{\text {rec }}\right)$ for a given BT; (B) $\mathrm{PV}^{\text {swept }}$ and $\mathrm{RF}^{\text {swept }}$ values for all time-steps, before and after BT (plain and dashed lines, respectively).
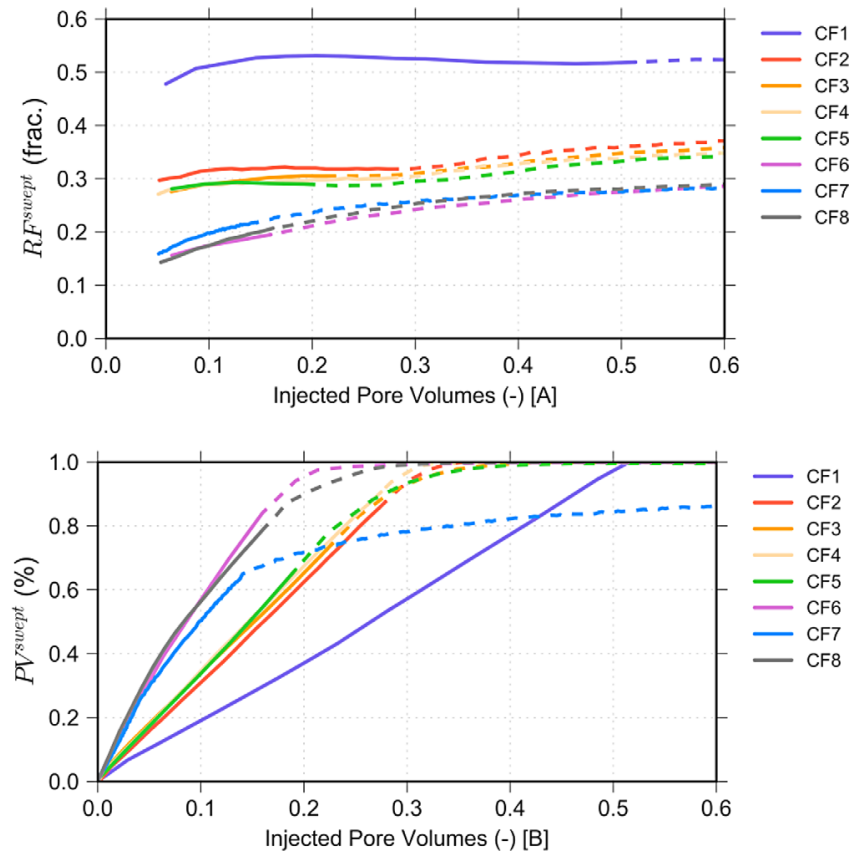

Fig. 21. $\mathrm{PV}^{\text {swept }}$ and $\mathrm{Mic}^{\mathrm{rec}}$ values measured at $\mathrm{BT}$ on the 3D CT-scans, as function of the number of PV injected. Plain lines show the values before BT, dashed lines show the values after BT.

sweeping during the flooding. For all corefloods except CF7, the sweeping efficiency reaches $100 \%$ quickly after BT (Fig. 21B). It shows that viscous fingering is not causing phase trapping unlike capillary trapping. For all corefloods, all the remaining oil is trapped by capillary forces after

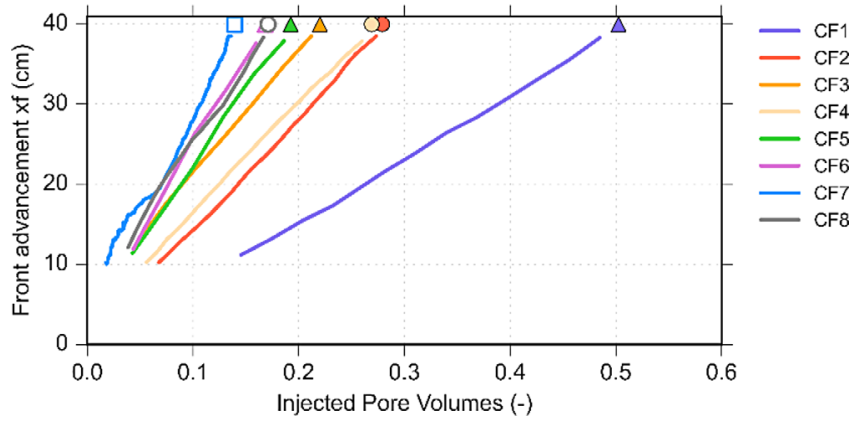

Fig. 22. Water-front advancement $x f$ as function of the number of pore-volumes injected. The markers show the oil-recovery at breakthrough (when $x f=40 \mathrm{~cm}$ ).

0.5 PV injected. Improvement of the oil-recovery is then solely caused by improvement of the microscopic recovery only (Fig. 21A). For the coreflood CF7, the $\mathrm{PV}^{\text {swept }}$ values don't reach $100 \%$ even after large number of PV injected. This phase trapping is not caused by the viscous digitations but by the gravity segregation.

The dynamic microscopic oil recovery curves (Fig. 21A) show specific trends for the two wettability systems. For the water-wet case (CF1-CF5), the RF ${ }^{\text {swept }}$ curves remain constant from the beginning of the injection (after stabilization) until BT. The oil recovery in swept areas only starts to improve when more than $90 \%$ of the core is swept. For the oil-wet case (CF6-CF8), it is improving since the beginning of the injection, log linearly with time. When expressed as function of the number of injected pore-volumes, they demonstrate a similar trend while the flowrate is changed by more than two decades for CF6 and CF7. This 
improvement of the oil-recovery during the flooding can be explained using the de Haan's pore-scale approach previously discussed [15]. The oil left in the core remains as a continuous phase through the pore restrictions and the grains coating. This phase connectivity enables a slow oil production and therefore a slow improvement of the microscopic oil recovery [37]. For the water-wet case, the oil is mostly trapped as disconnected ganglia and is therefore trapped by strong capillary forces. A substantial change of flowing conditions is needed to recover this oil, either by increasing the pressure gradient or lowering the capillary forces. The recover observed after BT can be explained using Buckley and Leverett theory [38], with a slow tail production occurring behind the water front in the swept areas.

These differences between the water-wet and the oil-wet cases lead to a different signature on the $\mathrm{PV}^{\text {swept }}$ and $\mathrm{RF}^{\text {swept }}$ cross-plot (Fig. 20B). For the water-wet corefloods, the vertical evolution of the curves shows the dissociation of the production mechanisms: first, oil is produced from unswept areas and secondly, it is produced from the swept areas. For the oil-wet corefloods, the curves are no longer vertical as oil is produced from both the swept and unswept areas during the flooding.

\section{Conclusion}

In this study, unstable displacements have been observed using dynamic CT-scan imaging. All displacements have been conducted in the same homogeneous Bentheimer core, under varying conditions of injection flowrate, displaced fluids viscosity and core wettability. All of these three contributions have demonstrated that they impact the digitation behavior.

First, the digitations have been characterized using the water breakthrough earliness. It showed a good correlation with the $N_{I}$ number as defined by Doorwar [11] for the water-wet case. A different scaling was observed for the oil-wet case. However, observations have suggested that the effect of the flowrate, the fluids viscosity and core wettability couldn't be considered individually, mitigating the possibility of constructing a unique scaling dimensionless number. For the water-case, higher injection flowrate is increasing the digitations, while it is no longer observed for the oil-wet case. It was found that the injection flowrate is not directly affecting the digitations itself, but it rather moderates the role of the capillary forces on the front stabilization, for the water-wet case.

Secondly, the local saturation monitoring has provided new insight to characterize the finger shapes and analyze the production mechanisms, for the different flowing conditions. We observed that the fingers pattern was dominated by the core wettability. For the water-case, the digitations take the form a several mains fingers growing at the center of the core. The latter is eventually merged in a main finger by viscous crossflow. For the oil-wet case, the viscous crossflow is dampened by the change of wettability. The fingers are developed at a smaller scale, the waterfront showed a less cohesive front shape. The local saturation monitoring allowed to distinguish two independent contributions to early breakthrough: viscous dominated digitations and capillary dominated digitations. A two-phases diagram has been constructed to plot and compare these contributions for all flowing conditions. Our results suggested that despite the clear front digitations, the early breakthrough was mostly caused by capillary fingering for all corefloods.

Finally, the evolution of these two contributions, computed for all time steps before and after breakthrough, shows the main production mechanisms during the flooding. Their evolutions show the main production mechanisms during the flooding. We observed that the macroscale digitations were not causing phase trapping at core scale: the core is completely swept after breakthrough. For the water-wet case, we found that the local oil recovery of swept zone remained constant before and after breakthrough while for the oil-wet case it is improving during all the water flooding.

Acknowledgments. Experiments have been conducted with the technical expertise of M.C. Lynch for the CT-scanner and F. Martin for the corefloods.

\section{References}

1 Dyes A.B., Caudle B.H., Erickson R.A. (1954) Oil production after breakthrough as influenced by mobility ratio, J. Petrol. Technol. 6, 04, 27-32. SPE-213-G. https://doi. org $/ 10.2118 / 309-G$.

2 van Meurs P., van der Poel C. (1958) A theoretical description of water-drive processes involving viscous fingering, Society of Petroleum Engineers.

3 Perkins T.K., Johnston O.C. (1969) A study of immiscible fingering in linear models, Soc. Pet. Eng. J. 9, 01, 39-46. SPE-8371-PA. https://doi.org/10.2118/2230-PA.

4 Hagoort J. (1974) Displacement stability of water drives in water-wet connate-water-bearing reservoirs, Soc. Pet. Eng. J. 14, 01, 63-74. SPE-8371-PA. https://doi.org/10.2118/ 4268-PA.

5 Yortsos Y.C., Huang A.B. (1986) Linear-stability analysis of immiscible displacement: Part 1-simple basic flow profiles, SPE Reserv. Eng. 1, 04, 378-390. SPE-12692-PA. https://doi.org/10.2118/12692-PA.

6 Koval E.J. (1963) A method for predicting the performance of unstable miscible displacement in heterogeneous media, Soc. Pet. Eng. J. 3, 02, 145-154. SPE-8371-PA. https://doi. org $/ 10.2118 / 450-P A$.

7 Rapoport L.A., Leas W.J. (1953) Properties of linear waterfloods, J. Petrol. Technol. 5, 05, 139-148. SPE-213-G. https://doi.org/10.2118/213-G.

8 Peters E.J., Flock D.L. (1981) The onset of instability during two-phase immiscible displacement in porous media, Soc. Pet. Eng. J. 21, 02, 249-258. SPE-8371-PA. https://doi.org/ 10.2118/8371-PA.

9 Chuoke R.L., van Meurs P., van der Poel C. (1959) The instability of slow, immiscible, viscous liquid-liquid displacements in permeable media, Society of Petroleum Engineers.

10 Lenormand R., Touboul E., Zarcone C. (1988) Numerical models and experiments on immiscible displacements in porous media, J. Fluid Mech. 189, 165-187. https://doi.org/ $10.1017 /$ S0022112088000953. 
11 Doorwar S. (2015) Understanding unstable immiscible displacement in porous media, The University of Texas at Austin, Austin, TX, USA. https://doi.org/10.15781/T25G6Q.

12 Bouquet S., Douarche F., Roggero F., Leray S. (2020) Characterization of viscous fingering and channeling for the assessment of polymer-based heavy oil displacements, Transp. Porous Media 131, 3, 873-906. https://doi.org/ 10.1007/s11242-019-01370-3.

13 Worawutthichanyakul T., Mohanty K.K. (2017) Unstable immiscible displacements in oil-wet rocks, Transp. Porous Media 119, 1, 205-223. https://doi.org/10.1007/s11242-0170880-6.

14 Vives M.T., Chang Y.C., Mohanty K.K. (1995) Effect of wettability on adverse mobility immiscible floods, Society of Petroleum Engineers, $10 \mathrm{p}$.

15 de Haan J. (1959) 25. Effect of Capillary Forces in the Water-Drive Process, World Petroleum Congress, 18 p.

16 Chatzis I., Dullien F. (1983) Dynamic immiscible displacement mechanisms in pore doublets: Theory versus experiment, J. Coll. Interf. Sci. 91, 1, 199-222. https://doi.org/ 10.1016/0021-9797(83)90326-0.

17 Loubens R.D., Vaillant G., Regaieg M., Yang J., Moncorgé A., Fabbri C., Darche G. (2017) Numerical modeling of unstable water floods and tertiary polymer floods into highly viscous oils, in: SPE Reservoir Simulation Conference, February 20-22, 2017, Montgomery, TX, USA, Society of Petroleum Engineers. https://doi.org/10.2118/182638-MS.

18 Doorwar S., Ambastha A. (2020) Pseudorelative permeabilities for simulation of unstable viscous oil displacement, $S P E$ Reserv. Eval. Eng. 23, 04, 1403-1419. SPE-179648-PA. https: //doi.org/10.2118/200421-PA.

19 Luo H., Mohanty K.K., Delshad M. (2017) Modeling and upscaling unstable water and polymer floods: Dynamic characterization of the effective viscous fingering, SPE Reserv. Eval. Eng. 20, 04, 779-794. SPE-179648-PA. https://doi.org/10.2118/179648-PA.

20 Doorwar S., Mohanty K.K. (2017) Viscous-fingering function for unstable immiscible flows (includes associated Erratum), SPE J. 22, 01, 19-31. SPE-173290-PA. https://doi.org/ 10.2118/173290-PA.

21 Fayers F.J. (1988) An approximate model with physically interpretable parameters for representing miscible viscous fingering, SPE Reserv. Eng. 3, 02, 551-558. SPE-12692-PA. https://doi.org/10.2118/13166-PA.

22 Sharma J., Inwood S.B., Kovscek A. (2012) Experiments and analysis of multiscale viscous fingering during forced imbibition, SPE J. 17, 04, 1142-1159. SPE-173290-PA. https://doi.org/10.2118/143946-PA.

23 Skauge A., Ormehaug P.A., Gurholt T., Vik B., Bondino I., Hamon G. (2012) 2-D visualisation of unstable waterflood and polymer flood for displacement of heavy oil, Society of Petroleum Engineers, 12 p.

24 Doorwar S., Mohanty K.K. (2014) Extension of the dielectric breakdown model for simulation of viscous fingering at finite viscosity ratios, Phys. Rev. E Stat. Nonlinear Soft Matter Phys. 90, 1, 13028. https://doi.org/10.1103/PhysRevE.90.013028.

25 Raeesi B., Morrow N.R., Mason G. (2014) Capillary pressure hysteresis behavior of three sandstones measured with a multistep outflow-inflow apparatus, Vadose Zone J. 13, 3. https://doi.org/10.2136/vzj2013.06.0097.

26 Lin Q., Bijeljic B., Pini R., Blunt M.J., Krevor S. (2018) Imaging and measurement of pore-scale interfacial curvature to determine capillary pressure simultaneously with relative permeability, Water Resour. Res. 54, 9, 7046-7060. https://doi.org/10.1029/2018WR023214.

27 Harleman D.R.F., Rumer R.R. (1963) Longitudinal and lateral dispersion in an isotropic porous medium, J. Fluid Mech. 16, 3, 385-394. https://doi.org/10.1017/S0022112063000847.

28 Brock D.C., Orr F.M. Jr (1991) Flow visualization of viscous fingering in heterogeneous porous media, Society of Petroleum Engineers, $12 \mathrm{p}$.

29 Riaz A., Tchelepi H.A. (2004) Linear stability analysis of immiscible two-phase flow in porous media with capillary dispersion and density variation, Phys. Fluids 16, 12, 47274737. https://doi.org/10.1063/1.1812511.

30 Anton L., Hilfer R. (1999) Trapping and mobilization of residual fluid during capillary desaturation in porous media, Phys. Rev. E Stat. Phys. Plasmas Fluids Relat. Interdiscip. Top. 59, 6, 6819-6823. https://doi.org/10.1103/physreve. 59.6819.

31 Berg S., Ott H. (2012) Stability of $\mathrm{CO}_{2}$ - brine immiscible displacement, Int. J. Greenhouse Gas Cont. 11, 188-203. https://doi.org/10.1016/j.ijggc.2012.07.001.

32 Pavone D. (1992) Observations and correlations for immiscible viscous-fingering experiments, SPE Reserv. Eng. 7, 02, 187-194. SPE-12692-PA. https://doi.org/10.2118/ 19670-PA.

33 Craig F.F. (1971) The reservoir engineering aspects of waterflooding, Society of Petroleum Engineers. ISBN: 978-089520-202-4.

34 Tang G.-Q., Kovscek A.R. (2011) High resolution imaging of unstable, forced imbibition in berea sandstone, Transp. Porous Media 86, 2, 617-634. https://doi.org/10.1007/ s11242-010-9643-3.

35 Mascle M., Youssef S., Deschamps H., Vizika O. (2019) In-situ investigation of aging protocol effect on relative permeability measurements using high-throughput experimentation methods, Petrophys. - SPWLA J. Form. Eval. Reserv. Description 60, 04, 514-524. SPWLA-2019-v60n4a5.

36 Clauset A., Shalizi C.R., Newman M.E.J. (2009) Power-law distributions in empirical data, SIAM Rev. 51, 4, 661-703. https://doi.org/10.1137/070710111.

37 Salathiel R.A. (1973) Oil recovery by surface film drainage in mixed-wettability rocks, J. Petrol. Technol. 25, 10, 12161224. SPE-213-G. https://doi.org/10.2118/4104-PA.

38 Buckley S.E., Leverett M.C. (1942) Mechanism of fluid displacement in sands, Trans. AIME 146, 01, 107-116. SPE942107-G. https://doi.org/10.2118/942107-G. 